\title{
Space regularity for evolution operators modeled on Hörmander vector fields with time dependent measurable coefficients
}

\author{
MARCO BRAMANTID
}

Abstract. We consider a heat-type operator $\mathcal{L}$ structured on the left invariant 1-homogeneous vector fields which are generators of a Carnot group, with a uniformly positive matrix of bounded measurable coefficients depending only on time. We prove that if $\mathcal{L} u$ is smooth with respect to the space variables, the same is true for $u$, with quantitative regularity estimates in the scale of Sobolev spaces defined by right invariant vector fields. Moreover, the solution and its space derivatives of every order satisfy a 1/2-Hölder continuity estimate with respect to time. The result is proved both for weak solutions and for distributional solutions, in a suitable sense.

Let $\mathbb{G}=\left(\mathbb{R}^{N}, \circ, D_{\lambda}\right)$ be a Carnot group and let $X_{1}, \ldots, X_{q}$ be the generators of its Lie algebra, so that the canonical sublaplacian

$$
\sum_{i=1}^{q} X_{i}^{2}
$$

and the corresponding heat operator

$$
\sum_{i=1}^{q} X_{i}^{2}-\partial_{t}
$$

are hypoelliptic in $\mathbb{R}^{N}$ and $\mathbb{R}^{N+1}$, respectively. (Precise definitions will be given in Sect. 1.) Let us now consider

$$
\mathcal{L}=\sum_{i, j=1}^{q} a_{i j}(t) X_{i} X_{j}-\partial_{t}
$$

where $\left\{a_{i j}(t)\right\}_{i, j=1}^{q}$ is a real, symmetric, uniformly positive matrix of bounded measurable coefficients:

$$
v|\xi|^{2} \leqslant \sum_{i, j=1}^{q} a_{i j}(t) \xi_{i} \xi_{j} \leqslant v^{-1}|\xi|^{2}
$$

Mathematics Subject Classification: Primary 35R03. Secondary 35B65. 35R05

Keywords: Carnot groups, Heat-type operators, Discontinuous coefficients, Hörmander's theorem. 
for some $v>0$, every $\xi \in \mathbb{R}^{q}$, a.e. $t \in(0, T)$. We want to prove a regularity result for $\mathcal{L}$ in the space variables, that is, roughly speaking: if $u \in W^{1,2}\left((0, T), L_{\text {loc }}^{2}\left(\mathbb{R}^{N}\right)\right)$ is a weak solution to $\mathcal{L} u=F, u(0, \cdot)=0$ and $F$ is smooth, with respect to the space variables, in some domain $(0, T) \times \Omega$, then the same is true for $u$, with quantitative regularity estimates on $u$ in terms of $\mathcal{L} u$. Also, we will prove that, if $F$ is smooth w.r.t. the space variables, then $u$ and every space derivative $\partial_{x}^{\alpha} u$ are $\frac{1}{2}$-Hölder continuous with respect to $t$. See Theorem 2.3 for the precise statements. This kind of regularity is the best we can hope, even for a uniformly parabolic operator

$$
\mathcal{L} u=u_{t}-a(t) u_{x x}
$$

as soon as $a$ is only $L^{\infty}$ (see Example 2.16). The above regularity result can be extended also to distributional solutions belonging to $W^{1,2}\left((0, T), \mathcal{D}^{\prime}\left(\mathbb{R}^{N}\right)\right.$ ) (see Theorem 3.3 for the precise statement). This can be seen as a kind of Hörmander's theorem with respect to the space variables.

A result of this kind has been proved by Krylov [12], who considered operators

$$
\mathcal{L}=\partial_{t}-\sum_{k=1}^{q} L_{k}^{2}+L_{0}
$$

with

$$
L_{k}=\sum_{i=1}^{N} \sigma^{i k}(t, x) \partial_{x_{i}}
$$

where the functions $\sigma^{i k}(t, x)$ are assumed to have $x$-derivatives of every order uniformly bounded for $x \in \mathbb{R}^{N}$ and $t \in(0,1)$, and the vector fields $L_{0}, L_{1}, \ldots, L_{q}$ for every fixed $t$ satisfy Hörmander's condition in $\mathbb{R}^{N}$. Now, every operator $(0.1)$ can be rewritten as

$$
-\mathcal{L}=\partial_{t}-\sum_{k=1}^{q} L_{k}^{2}
$$

with

$$
\sigma^{i k}(t, x)=\sum_{j=1}^{q} m_{j k}(t) b_{j i}(x)
$$

where

$$
X_{j}=\sum_{i=1}^{N} b_{j i}(x) \partial_{x_{i}}
$$

and

$$
a_{i j}(t)=\sum_{k=1}^{q} m_{i k}(t) m_{j k}(t)
$$


so that

$$
\left|D_{x}^{\alpha} \sigma^{i k}(t, x)\right| \leqslant c_{\nu} \sum_{j=1}^{q}\left|D_{x}^{\alpha} b_{j i}(x)\right| .
$$

Since the coefficients $b_{j i}(x)$ of the generators on a Carnot group are polynomials, the functions $\left|D_{x}^{\alpha} b_{j i}(x)\right|$ are not globally bounded on $\mathbb{R}^{N}$. Therefore, although the class of operators that we consider is strictly contained in the class considered by Krylov as to their structure, the assumption on $\sigma^{i k}(t, x)$ made in [12] is not satisfied in our situation.

Actually, the technique employed in this paper is very different from that in [12]. In [12], following the classical approach introduced by Kohn [11] and Oleĭnik-Radkevič [15], pseudodifferential operators and Sobolev spaces of fractional order are used. Here, instead, we adapt to the evolutionary case the technique introduced in [2] to give a proof of Hörmander's theorem for sublaplacians on Carnot groups. The main idea consists in measuring the regularity of solutions of an equation $\mathcal{L} u=f$, where $\mathcal{L}$ is a left invariant operator, in terms of Sobolev spaces induced by right invariant vector fields. Since a right invariant operator and a left invariant operator always commute, this approach greatly simplifies the proof of higher-order estimates. We handle Sobolev norms with respect to vector fields by means of equivalent norms defined in terms of finite difference operators, in the directions of the vector fields $X_{1}, \ldots, X_{q}$. This feature of our argument is reminiscent of the original proof of Hörmander's theorem given in [10], although in the richer framework of Carnot groups the proof becomes much simpler.

Let us now give some motivation for the present research and describe some related literature. The regularity result proved in [12] has been applied by the same Author in [13] to prove an analogous result for stochastic PDEs, and in [14], in the context of filtering problems. We refer to [13] for motivations to prove this result without any continuity assumption on the coefficients with respect to time.

Hyperbolic operators of the kind

$$
H u=u_{t t}-\sum_{i, j=1}^{n} a_{i j}(t) u_{x_{i} x_{j}}
$$

with merely bounded measurable $a_{i j}$ have been studied by many authors, see, for instance, $[6,7,9]$ and references therein. In particular, [9] gives some physical motivation to study this class of operators under no regularity condition on $a_{i j}(t)$.

Operators of the kind

$$
\mathcal{L}=\sum_{i, j=1}^{q} a_{i j}(t, x) X_{i} X_{j}-\partial_{t},
$$

satisfying (0.2) have been studied by several Authors, assuming the coefficients $a_{i j}(t, x)$ either Hölder continuous or with vanishing mean oscillation, and proving a priori estimates and regularity results in the scale of Hölder or Sobolev spaces induced by 
the vector fields $\left\{X_{i}\right\}_{i=1}^{q}$ and the distance they induce. See, for instance, [3-5] and references therein. In [4], for the operator $\mathcal{L}$ with Hölder continuous coefficients, a heat kernel has been constructed and shown to satisfy sharp Gaussian estimates, which also imply a scale invariant Harnack inequality.

The operators $(0.1)$ studied in the present paper can also be seen as model operators to study the more general class $(0.3)$ with the coefficients satisfying some moderate regularity assumption in $x$, but only $L^{\infty}$ with respect to time, an area of research that we plan to attack in the future.

\section{Preliminaries about Carnot groups}

Let us recall some standard definitions and results that will be useful in the following. For the proofs of these facts, the reader is referred to [8], [1, Chap.1]. A homogeneous group (in $\mathbb{R}^{N}$ ) is a Lie group $\left(\mathbb{R}^{N}, \circ\right)$ (where the group operation $\circ$ will be thought as a "translation") endowed with a one parameter family $\left\{D_{\lambda}\right\}_{\lambda>0}$ of group automorphisms ("dilations") which act this way:

$$
D_{\lambda}\left(x_{1}, x_{2}, \ldots, x_{N}\right)=\left(\lambda^{\alpha_{1}} x_{1}, \lambda^{\alpha_{2}} x_{2}, \ldots, \lambda^{\alpha_{N}} x_{N}\right)
$$

for suitable integers $1=\alpha_{1} \leqslant \alpha_{2} \leqslant \cdots \leqslant \alpha_{N}$. We will write $\mathbb{G}=\left(\mathbb{R}^{N}, \circ, D_{\lambda}\right)$ to denote this structure. The number

$$
Q=\sum_{i=1}^{N} \alpha_{i}
$$

will be called homogeneous dimension of $\mathbb{G}$. A homogeneous norm on $\mathbb{G}$ is a continuous function

$$
\|\cdot\|: \mathbb{G} \rightarrow[0,+\infty)
$$

such that, for some constant $c>0$ and every $x, y \in \mathbb{G}$,

$$
\begin{aligned}
& \text { (i) }\|x\|=0 \Longleftrightarrow x=0 \\
& \text { (ii) }\left\|D_{\lambda}(x)\right\|=\lambda\|x\| \forall \lambda>0 \\
& \text { (iii) }\left\|x^{-1}\right\| \leqslant c\|x\| \\
& \text { (iv) }\|x \circ y\| \leqslant c(\|x\|+\|y\|) .
\end{aligned}
$$

We will always use the symbol $\|\cdot\|$, without any subscript, to denote a homogeneous norm in $\mathbb{G}$. Examples of homogeneous norms are the following:

$$
\|x\|=\max _{k=1,2, \ldots, N}\left|x_{k}\right|^{\frac{1}{\alpha_{k}}}
$$

or

$$
\|x\|=\left(\sum_{k=1}^{N}\left|x_{k}\right|^{\frac{Q}{\alpha_{k}}}\right)^{1 / Q} .
$$


It can be proved that any two homogeneous norms on $\mathbb{G}$ are equivalent.

We say that a smooth function $f$ in $\mathbb{G} \backslash\{0\}$ is $D_{\lambda}$-homogeneous of degree $\beta \in \mathbb{R}$ (or simply " $\beta$-homogeneous") if

$$
f\left(D_{\lambda}(x)\right)=\lambda^{\beta} f(x) \quad \forall \lambda>0, x \in \mathbb{G} \backslash\{0\} .
$$

Given any differential operator $P$ with smooth coefficients on $\mathbb{G}$, we say that $P$ is left invariant if for every $x, y \in \mathbb{G}$ and every smooth function $f$

$$
P\left(L_{y} f\right)(x)=L_{y}(P f(x)),
$$

where

$$
L_{y} f(x)=f(y \circ x) .
$$

Analogously, one defines the notion of right invariant differential operator. Also, $P$ is said $\beta$-homogeneous (for some $\beta \in \mathbb{R}$ ) if

$$
P\left(f\left(D_{\lambda}(x)\right)\right)=\lambda^{\beta}(P f)\left(D_{\lambda}(x)\right)
$$

for every smooth function $f, \lambda>0$ and $x \in \mathbb{G}$.

A vector field is a first-order differential operator

$$
X=\sum_{i=1}^{N} c_{i}(x) \partial_{x_{i}} .
$$

Let $\mathfrak{g}$ be the Lie algebra of left invariant vector fields over $\mathbb{G}$, where the Lie bracket of two vector fields is defined as usual by

$$
[X, Y]=X Y-Y X .
$$

Let us denote by $X_{1}, X_{2}, \ldots, X_{N}$ the canonical base of $\mathfrak{g}$, that is for $i=1,2, \ldots, N$, $X_{i}$ is the only left invariant vector field that agrees with $\partial_{x_{i}}$ at the origin. Also, $X_{1}^{R}, X_{2}^{R}, \ldots, X_{N}^{R}$ will denote the right invariant vectors fields that agree with $\partial_{x_{1}}, \partial_{x_{2}}, \ldots, \partial_{x_{N}}$ (and hence with $X_{1}, X_{2}, \ldots, X_{N}$ ) at the origin.

We assume that for some integer $q<N$, the vector fields $X_{1}, X_{2}, \ldots, X_{q}$ are 1homogeneous and the Lie algebra generated by them is $\mathfrak{g}$. If $s$ is the maximum length of commutators

$$
\left[X_{i_{1}},\left[X_{i_{2}}, \ldots,\left[X_{i_{s-1}}, X_{i_{s}}\right]\right]\right], i_{j} \in\{1,2, \ldots, q\}
$$

required to span $\mathfrak{g}$, then we will say that $\mathfrak{g}$ is a stratified Lie algebra of step $s, \mathbb{G}$ is a Carnot group (or a stratified homogeneous group) and its generators $X_{1}, X_{2}, \ldots, X_{q}$ satisfy Hörmander's condition at step $s$ in $\mathbb{G}$. Under these assumptions, by Hörmander's theorem (see [10]), the canonical sublaplacian

$$
L=\sum_{i=1}^{q} X_{i}^{2}
$$


is hypoelliptic in $\mathbb{R}^{N}$, that is: for every domains $\Omega^{\prime} \subset \Omega \subset \mathbb{R}^{N}$, whenever $u \in \mathcal{D}^{\prime}(\Omega)$ solves in distributional sense the equation $L u=f$ in $\Omega$, then $f \in C^{\infty}\left(\Omega^{\prime}\right) \Rightarrow u \in$ $C^{\infty}\left(\Omega^{\prime}\right)$.

Analogously, the corresponding heat operator

$$
H=\sum_{i=1}^{q} X_{i}^{2}-\partial_{t}
$$

is hypoelliptic in $\mathbb{R}^{N+1}$.

The following compact notation will be used throughout the following. For a multiindex

$$
I=\left(i_{1}, \ldots, i_{k}\right), i_{j} \in\{1,2, \ldots, q\}
$$

we let

$$
\begin{aligned}
X_{I} & =X_{i_{1}} X_{i_{2}} \ldots X_{i_{k}} \\
X_{[I]} & =\left[X_{i_{1}},\left[X_{i_{2}}, \ldots,\left[X_{i_{k-1}}, X_{i_{k}}\right]\right]\right] \\
|I| & =k
\end{aligned}
$$

with an analogous meaning for $X_{I}^{R}, X_{[I]}^{R}$.

We will make use of the Sobolev spaces $W_{X}^{k, p}(\mathbb{G}), W_{X^{R}}^{k, p}(\mathbb{G})$ induced by the systems of vector fields

$$
X=\left\{X_{1}, X_{2}, \ldots, X_{q}\right\}, X^{R}=\left\{X_{1}^{R}, X_{2}^{R}, \ldots, X_{q}^{R}\right\},
$$

respectively. More precisely, given an open subset $\Omega$ of $\mathbb{R}^{N}$, we say that $f \in W_{X}^{k, 2}(\Omega)$ if $f \in L^{2}(\Omega)$ and there exist, in weak sense, $X_{I} f \in L^{2}(\Omega)$ for every multiindex $I$ with $|I| \leq k$. We set

$$
\|f\|_{W_{X}^{k, 2}(\Omega)}=\|f\|_{L^{2}(\Omega)}+\sum_{|I| \leq k}\left\|X_{I} f\right\|_{L^{2}(\Omega)} .
$$

The space $W_{X^{R}}^{k, 2}(\Omega)$ has a similar definition. We will also use local Sobolev spaces. For example, we will say that $f \in W_{X, \text { loc }}^{k, 2}(\Omega)$ if for every $\varphi \in C_{0}^{\infty}(\Omega)$, we have $\varphi f \in W_{X}^{k, 2}(\Omega)$.

For homogeneity reasons, the generators $X_{1}, \ldots, X_{q}$ satisfy the simple relation $X_{i}^{*}=-X_{i}$ (where $X^{*}$ stands for the transposed operator of $X$ ). In other words,

$$
\int_{\mathbb{G}} f\left(X_{i} g\right)=-\int_{\mathbb{G}}\left(X_{i} f\right) g
$$

whenever $f \in W_{X, \text { loc }}^{1,2}(\mathbb{G})$ and $g \in C_{0}^{1}(\mathbb{G})$.

The validity of Hörmander's condition at step $s$ implies the following important: 
Proposition 1.1. (See [2, Prop. 2.1]) Under the above assumptions we have:

1.

$$
\bigcap_{k=1}^{\infty} W_{X}^{k, 2}(\Omega) \subset C^{\infty}(\Omega) .
$$

2. For any positive integer $k$ and any $\Omega^{\prime} \Subset \Omega$ there exists a constant $c>0$ such that, for every $u \in W_{X}^{k s, 2}(\Omega)$ we have

$$
\|u\|_{W^{k, 2}\left(\Omega^{\prime}\right)} \leqslant c\|u\|_{W_{X}^{k s, 2}(\Omega)},
$$

where $W^{k, 2}\left(\Omega^{\prime}\right)$ denotes the standard Sobolev space. Analogous properties hold for the spaces $W_{X^{R}}^{k, 2}(\Omega)$.

Let us point out a relation between left and right invariant operators which will be very useful in the following.

Proposition 1.2. (see [2, Prop. 2.2]) Let $\mathcal{L}, \mathcal{R}$ be any two differential operators on $\mathbb{G}$ with smooth coefficients, left and right invariant, respectively. Then, $\mathcal{L}$ and $\mathcal{R}$ commute:

$$
\mathcal{L} \mathcal{R} f=\mathcal{R} \mathcal{L} f
$$

for any smooth function $f$.

For every given couple of measurable functions $\varphi, \psi: \mathbb{G} \rightarrow \mathbb{R}$, we define

$$
\varphi * \psi(x)=\int_{\mathbb{G}} \varphi(y) \psi\left(y^{-1} \circ x\right) \mathrm{d} y
$$

whenever the integral makes sense. One can prove the following:

Proposition 1.3. For every couple of measurable functions $f, \psi$ defined on $\mathbb{G}$ such that the following convolutions are well defined, we have

i) if $\mathcal{P}$ is a left invariant differential operator then

$$
\mathcal{P}(f * \psi)=f * \mathcal{P} \psi,
$$

ii) if $\mathcal{P}$ is a right invariant differential operator then

$$
\mathcal{P}(\psi * f)=\mathcal{P} \psi * f
$$

whenever $\mathcal{P} \psi$ exists at least in weak sense. 


\section{Subelliptic estimates for heat-type operators with $t$-measurable coefficients}

For a domain $\Omega \subseteq \mathbb{G}$, let

$$
\Omega_{T}=(0, T) \times \Omega .
$$

We are going to define several function spaces on $\mathbb{G}_{T}=(0, T) \times \mathbb{G}$ that we will use in the following.

The definitions of the spaces $L^{2}((0, T), X), W^{1,2}((0, T), X), C^{0}([0, T], X)$ when $X$ is a Banach space are standard. For instance, we will often use the spaces

$$
L^{2}\left((0, T), W_{X}^{k, 2}(\mathbb{G})\right)
$$

(for $k=1,2,3, \ldots$ ) normed with

$$
\|f\|_{L^{2}\left((0, T), W_{X}^{k, 2}(\mathbb{G})\right)}=\|f\|_{L^{2}\left(\mathbb{G}_{T}\right)}+\sum_{|I| \leq k}\left\|X_{I} f\right\|_{L^{2}\left(\mathbb{G}_{T}\right)}
$$

and the analogous spaces $L^{2}\left((0, T), W_{X^{R}}^{k, 2}(\mathbb{G})\right)$.

We will say that $u \in L^{2}\left((0, T), W_{X, \text { loc }}^{k, 2}(\mathbb{G})\right)$ when for every $\zeta \in C_{0}^{\infty}(\mathbb{G})$ we have $u \zeta \in L^{2}\left((0, T), W_{X}^{k, 2}(\mathbb{G})\right)$.

For a function $f \in L^{2}\left((0, T), W_{X}^{1,2}(\mathbb{G})\right)$, we will also use the shorthand notation

$$
\left\|\nabla_{X} f\right\|_{L^{2}\left(\mathbb{G}_{T}\right)}^{2}=\sum_{i=1}^{q}\left\|X_{i} f\right\|_{L^{2}\left(\mathbb{G}_{T}\right)}^{2}
$$

with the analogous meaning for $\left\|\nabla_{X^{R}} f\right\|_{L^{2}\left(\mathbb{G}_{T}\right)}^{2}$.

Definition 2.1. (Weak solution) We say that $u \in W^{1,2}\left((0, T), L_{\text {loc }}^{2}(\mathbb{G})\right)$ is a weak solution to $\mathcal{L} u=F \in L^{2}\left((0, T), L_{\text {loc }}^{2}(\mathbb{G})\right)$ if

$$
\int_{\mathbb{G}}\left\{-\partial_{t} u(t, x) \phi(x)+\sum_{i, j=1}^{q} a_{i j}(t) X_{i} X_{j} \phi(x) u(t, x)\right\} \mathrm{d} x=\int_{\mathbb{G}} F(t, x) \phi(x) \mathrm{d} x
$$

for every $\phi \in C_{0}^{\infty}(\mathbb{G})$ and a.e. $t \in(0, T)$.

Note that $W^{1,2}\left((0, T), L_{\text {loc }}^{2}(\mathbb{G})\right) \subset C^{0}\left([0, T], L_{\text {loc }}^{2}(\mathbb{G})\right)$, hence the initial value $u(0, \cdot)$ is a well defined element of $L_{\text {loc }}^{2}(\mathbb{G})$.

Definition 2.2. We say that a function $u$ belongs to $L^{2}\left((0, T), C^{\infty}(\bar{\Omega})\right)$ if $u \in$ $L^{2}\left((0, T), C^{k}(\bar{\Omega})\right)$ for every $k=0,1,2, \ldots$ In particular, this implies that

$$
\int_{0}^{T}\|u(t, \cdot)\|_{C^{k}(\bar{\Omega})}^{2} \mathrm{~d} t<\infty \text { for every } k=0,1,2, \ldots
$$


We say that a function $u$ belongs to $C^{0}\left([0, T], C^{\infty}(\bar{\Omega})\right)$ if $u \in C^{0}\left([0, T], C^{k}(\bar{\Omega})\right)$ for every $k=0,1,2, \ldots$

We can now state the first main result of this paper:

Theorem 2.3. Let $\mathbb{G}$ be a Carnot group of step $s$ with generators $X_{1}, \ldots, X_{q}$, let $\mathcal{L}$ be as in (0.1) and let $(0.2)$ be in force. Let $u \in W^{1,2}\left((0, T), L_{\mathrm{loc}}^{2}(\mathbb{G})\right), u(0, \cdot)=0$, be a weak solution to $\mathcal{L} u=F \in L^{2}\left((0, T), L_{\text {loc }}^{2}(\mathbb{G})\right)$ in the sense of $(2.1)$.

(i) For every $k=1,2,3, \ldots$, let $\zeta, \zeta_{1} \in C_{0}^{\infty}(\mathbb{G}), \zeta \prec \zeta_{1}$. There exists $c=$ $c\left(k, \zeta, \zeta_{1}, \mathbb{G}, v\right)>0$ such that

$$
\text { if } \zeta_{1} F \in L^{2}\left((0, T), W_{X^{R}}^{k+s^{2}-1,2}(\mathbb{G})\right) \text { then } \zeta u \in L^{2}\left((0, T), W_{X^{R}}^{k, 2}(\mathbb{G})\right)
$$

and

$$
\|\zeta u\|_{L^{2}\left((0, T), W_{X}^{k, 2}(\mathbb{G})\right)} \leqslant c\left\{\left\|\zeta_{1} F\right\|_{L^{2}\left((0, T), W_{X}^{k+s-1,2}(\mathbb{G})\right)}+\left\|\zeta_{1} u\right\|_{L^{2}\left(\mathbb{G}_{T}\right)}\right\} .
$$

(ii) In particular, if $F \in L^{2}\left((0, T), W_{X^{R}, \text { loc }}^{k+s^{2}+2 s-1,2}(\mathbb{G})\right)$ for some $k=0,1,2,3, \ldots$, then $u$ is also a strong solution to $\mathcal{L} u=F$ and for every multiindex $I$ with $|I| \leqslant k$ we have

$$
X_{I}^{R} u \in C^{0}\left([0, T], L_{\mathrm{loc}}^{2}(\mathbb{G})\right) \text { and } X_{I}^{R} u(0, \cdot)=0 .
$$

(iii) For every (cartesian) derivative $\partial_{x}^{\alpha}$ and $\zeta, \zeta_{1} \in C_{0}^{\infty}(\mathbb{G}), \zeta \prec \zeta_{1}$, there exists $c=c\left(\alpha, \zeta, \zeta_{1}, \mathbb{G}, v\right)>0$ and a positive integer $h$ such that whenever $F \in$ $L^{2}\left((0, T), W_{X^{R}, \text { loc }}^{h, 2}(\mathbb{G})\right)$ then

$$
\begin{aligned}
& \sup _{0<t_{1}<t_{2}<T} \sup _{x \in \mathbb{G}} \frac{\left|\zeta(x)\left[\partial_{x}^{\alpha} u\left(t_{2}, x\right)-\partial_{x}^{\alpha} u\left(t_{1}, x\right)\right]\right|}{\left|t_{2}-t_{1}\right|^{1 / 2}} \\
& \quad \leqslant c\left\{\left\|\zeta_{1} F\right\|_{L^{2}\left((0, T), W_{X^{R}}^{h, 2}(\mathbb{G})\right)}+\left\|\zeta_{1} u\right\|_{L^{2}\left(\mathbb{G}_{T}\right)}\right\}
\end{aligned}
$$

and

$$
\sup _{x \in \mathbb{G}}\left|\zeta(x) \partial_{x}^{\alpha} u(t, x)\right| \leqslant c|t|^{1 / 2}\left\{\left\|\zeta_{1} F\right\|_{L^{2}\left((0, T), W_{X}^{h, 2}(\mathbb{G})\right)}+\left\|\zeta_{1} u\right\|_{L^{2}\left(\mathbb{G}_{T}\right)}\right\} \forall t \in[0, T] .
$$

(iv) In particular, if

$$
\zeta_{1} F \in L^{2}\left((0, T), C^{\infty}(\mathbb{G})\right)
$$

then

$$
\zeta u \in C^{0}\left([0, T], C^{\infty}(\mathbb{G})\right) \text { and } \zeta u_{t} \in L^{2}\left((0, T), C^{\infty}(\mathbb{G})\right)
$$

The first step of the proof is a standard energy estimate. Before proving it, we need to define some more function spaces: 
Definition 2.4. We let:

$$
\begin{aligned}
\mathcal{H} & =L^{2}\left((0, T), W_{X}^{2,2}(\mathbb{G})\right) \cap W^{1,2}\left((0, T), L^{2}(\mathbb{G})\right) \\
& =\left\{u \in L^{2}\left(\mathbb{G}_{T}\right): u_{t}, X_{i} u, X_{i} X_{j} u \in L^{2}\left(\mathbb{G}_{T}\right)\right\} .
\end{aligned}
$$

Note that $\mathcal{H} \subset C^{0}\left([0, T], L^{2}(\mathbb{G})\right)$, so that for $u \in \mathcal{H}$ and $t \in[0, T], u(t, \cdot)$ is a well defined element of $L^{2}(\mathbb{G})$.

We will also use

$\mathcal{H}_{0}=\left\{u \in W^{1,2}\left((0, T), L_{\text {loc }}^{2}(\mathbb{G})\right): \forall \phi \in C_{0}^{\infty}(\mathbb{G}) u \phi \in \mathcal{H}\right.$ and $\left.(u \phi)(0, \cdot)=0\right\}$.

Proposition 2.5. Under the previous assumption on $\mathcal{L}$, for every $u \in \mathcal{H}$ such that $u(0, \cdot)=0$ we have

$$
\left\|\nabla_{X} u\right\|_{L^{2}\left(\mathbb{G}_{T}\right)} \leqslant c_{\nu}\left\{\|\mathcal{L} u\|_{L^{2}\left(\mathbb{G}_{T}\right)}+\|u\|_{L^{2}\left(\mathbb{G}_{T}\right)}\right\}
$$

for a constant $c_{\nu}$ only depending on the ellipticity constant $v$ in (0.2).

Proof. For $u \in \mathcal{H}$ we have, recalling that $X_{i}^{*}=-X_{i}$ (see (1.2)):

$$
\begin{gathered}
-\iint_{\mathbb{G}_{T}}(u \mathcal{L} u) \mathrm{d} t \mathrm{~d} x=\iint_{\mathbb{G}_{T}}\left(u \partial_{t} u\right) \mathrm{d} t \mathrm{~d} x-\iint_{\mathbb{G}_{T}}\left(u \sum_{i, j=1}^{q} a_{i j}(t) X_{i} X_{j} u\right) \mathrm{d} t \mathrm{~d} x \\
=\frac{1}{2} \int_{\mathbb{G}}\left(\int_{0}^{T} \partial_{t}\left(u^{2}\right) \mathrm{d} t\right) \mathrm{d} x-\sum_{i, j=1}^{q} \int_{0}^{T} a_{i j}(t)\left(\int_{\mathbb{G}}\left(u X_{i} X_{j} u\right) \mathrm{d} x\right) \mathrm{d} t \\
=\frac{1}{2} \int_{\mathbb{G}}\left(u^{2}(T, x)-u^{2}(0, x)\right) \mathrm{d} x+\sum_{i, j=1}^{q} \int_{0}^{T} a_{i j}(t)\left(\int_{\mathbb{G}}\left(X_{i} u X_{j} u\right) \mathrm{d} x\right) \mathrm{d} t .
\end{gathered}
$$

Since

$$
\sum_{i, j=1}^{q} \int_{0}^{T} a_{i j}(t)\left(\int_{\mathbb{G}}\left(X_{i} u X_{j} u\right) \mathrm{d} x\right) \mathrm{d} t \geq v \sum_{i=1}^{q} \int_{0}^{T} \int_{\mathbb{G}}\left(X_{i} u\right)^{2} \mathrm{~d} x \mathrm{~d} t
$$

we have

$$
\left\|\nabla_{X} u\right\|_{L^{2}\left(\mathbb{G}_{T}\right)}^{2} \leqslant \frac{1}{v}\|\mathcal{L} u\|_{L^{2}\left(\mathbb{G}_{T}\right)}\|u\|_{L^{2}\left(\mathbb{G}_{T}\right)}+\frac{1}{2 v}\|u(0, \cdot)\|_{L^{2}(\mathbb{G})} .
$$

In particular, for $u$ vanishing on $t=0$ we get (2.3).

In the following of this section, we will recall and adapt several definitions and arguments taken from [2]. The reader is referred to that paper for some details. 
Definition 2.6. (Finite difference operators) For every $h \in \mathbb{G}$ and function $f$ defined in $\mathbb{G}$, let us define the operators:

$$
\begin{aligned}
& \Delta_{h} f(x)=f(x \circ h)-f(x) \\
& \widetilde{\Delta}_{h} f(x)=f(h \circ x)-f(x) .
\end{aligned}
$$

Whenever the function $f$ also depends on $t$, we will simply write

$$
\Delta_{h} f(t, x)=\Delta_{h}[f(t, \cdot)](x)
$$

and analogously for $\widetilde{\Delta}_{h} f(t, x)$.

Definition 2.7. For $m=1,2,3,4, \ldots$, let

$$
\begin{aligned}
\Delta_{h}^{m} & =\underbrace{\Delta_{h} \Delta_{h} \cdots \Delta_{h}}_{m \text { times }} . \\
\widetilde{\Delta}_{h}^{m} & =\underbrace{\widetilde{\Delta}_{h} \widetilde{\Delta}_{h} \cdots \widetilde{\Delta}_{h}}_{m \text { times }} .
\end{aligned}
$$

Then, for $\alpha>0$ and $f \in L^{2}\left(\mathbb{G}_{T}\right)$ we define the semi-norms

$$
\begin{aligned}
& |f|_{m, \alpha}=\sup \left\{\frac{\left\|\Delta_{h}^{m} f\right\|_{L^{2}\left(\mathbb{G}_{T}\right)}}{\|h\|^{\alpha}}: h=\operatorname{Exp}\left(t X_{i}\right) \forall i=1, \ldots, q, t \in \mathbb{R}: 0<\|h\| \leqslant 1\right\} \\
& |f|_{m, \alpha}^{R}=\sup \left\{\frac{\left\|\widetilde{\Delta}_{h}^{m} f\right\|_{L^{2}\left(\mathbb{G}_{T}\right)}}{\|h\|^{\alpha}}: h=\operatorname{Exp}\left(t X_{i}\right) \forall i=1, \ldots, q, t \in \mathbb{R}: 0<\|h\| \leqslant 1\right\} .
\end{aligned}
$$

We also set for convenience

$$
\begin{aligned}
|f|_{0} & =|f|_{0}^{R}=\|f\|_{L^{2}\left(\mathbb{G}_{T}\right)} \\
|f|_{m} & =|f|_{m, m} \\
|f|_{m}^{R} & =|f|_{m, m}^{R} .
\end{aligned}
$$

The relations between the above seminorms and Sobolev norms with respect to vector fields are contained in the following two results, which can be derived by [2, Thm. 3.11, Prop.3.13] simply integrating in $t$.

Proposition 2.8. For $m=1,2, \ldots$ there exists $c=c(m, \mathbb{G})$ such that, for every $f \in L^{2}\left(\mathbb{G}_{T}\right)$ we have:

$$
\text { 1. If } f \in L^{2}\left((0, T), W_{X}^{m, 2}(\mathbb{G})\right) \text { then }
$$

$$
\sum_{k=0}^{m}|f|_{k} \leqslant c\|f\|_{L^{2}\left((0, T), W_{X}^{m, 2}(\mathbb{G})\right)}
$$

Analogously, 
2. If $f \in L^{2}\left((0, T), W_{X^{R}}^{m, 2}(\mathbb{G})\right)$ then

$$
\sum_{k=0}^{m}|f|_{k}^{R} \leqslant c\|f\|_{L^{2}\left((0, T), W_{X^{R}}^{m, 2}(\mathbb{G})\right)} .
$$

Proposition 2.9. There exists $C=C(\mathbb{G})$ such that for every $f \in L^{2}\left(\mathbb{G}_{T}\right)$ we have: 1. If $|f|_{1}<\infty$ then $f \in L^{2}\left((0, T), W_{X}^{1,2}(\mathbb{G})\right)$, with

$$
\left\|\nabla_{X} f\right\|_{L^{2}\left(\mathbb{G}_{T}\right)} \leqslant C|f|_{1} .
$$

2. If $|f|_{1}^{R}<\infty$ then $f \in L^{2}\left((0, T), W_{X^{R}}^{1,2}(\mathbb{G})\right)$, with

$$
\left\|\nabla_{X^{R}} f\right\|_{L^{2}\left(\mathbb{G}_{T}\right)} \leqslant C|f|_{1}^{R} .
$$

The following bound instead links the $L^{2}\left((0, T), W_{X}^{1,2}(\mathbb{G})\right)$ norm with the operators $\widetilde{\Delta}_{h}$ :

Proposition 2.10. Let $\Omega$ be a bounded domain in $\mathbb{G}$. There exists $c=c(\Omega, \mathbb{G})$ such that for every $u \in L^{2}\left((0, T), W_{X}^{1,2}(\mathbb{G})\right)$ with $\operatorname{sprt} u(t, \cdot) \subset \Omega$ for every $t \in(0, T)$ we have

$$
\left\|\widetilde{\Delta}_{h} u\right\|_{L^{2}\left(\mathbb{G}_{T}\right)} \leqslant c\|h\|^{1 / s}\left\|\nabla_{X} u\right\|_{L^{2}\left(\mathbb{G}_{T}\right)} .
$$

(Recall that s is the step of the Lie algebra.)

Proof. It is enough to apply to $u(t, \cdot)$ the computations made in [2, Prop.3.7, Lemma 3.8] for functions in $W_{X}^{1,2}(\mathbb{G})$ and then integrate on $(0, T)$.

If $u \in \mathcal{H}, u(t, \cdot)$ is supported in some bounded domain $\Omega$ for every $t \in[0, T]$ and $u(0, \cdot)=0$, then by the previous Proposition and $(2.3)$ we get

$$
\left\|\widetilde{\Delta}_{h} u\right\|_{L^{2}\left(\mathbb{G}_{T}\right)} \leqslant c_{\nu}\|h\|^{1 / s}\left\{\|\mathcal{L} u\|_{L^{2}\left(\mathbb{G}_{T}\right)}+\|u\|_{L^{2}\left(\mathbb{G}_{T}\right)}\right\}
$$

that is

$$
|u|_{1,1 / s}^{R} \leqslant c_{\nu}\left\{\|\mathcal{L} u\|_{L^{2}\left(\mathbb{G}_{T}\right)}+\|u\|_{L^{2}\left(\mathbb{G}_{T}\right)}\right\} .
$$

Notation 2.11. Henceforth, we will write

$$
\zeta_{0} \prec \zeta
$$

if $\zeta_{0}, \zeta \in C_{0}^{\infty}(\mathbb{G})$ such that $0 \leqslant \zeta_{0} \leqslant \zeta \leqslant 1$ and $\zeta=1$ on sprt $\zeta_{0}$.

We have the following analog of Theorem 3.15 in [2]: 
Theorem 2.12. Let $\zeta_{0}, \zeta \in C_{0}^{\infty}(\mathbb{G})$ with $\zeta_{0} \prec \zeta$. For every $m \in \mathbb{N}$ the exists $c=$ $c\left(\zeta_{0}, \zeta, m, \mathbb{G}, v\right)>0$ such that if $u \in \mathcal{H}_{0}$ then

$$
\left|\zeta_{0} u\right|_{m, m / s}^{R} \leqslant c\left(\sum_{j=0}^{m-1}|\zeta \mathcal{L} u|_{j}^{R}+\|\zeta u\|_{2}\right)
$$

whenever the right-hand side is finite.

Proof. We can repeat the proof of Theorem 3.15 in [2] applying (2.8) to the function $\zeta_{0} u \in \mathcal{H}$, since $u \in \mathcal{H}_{0}$, and exploiting the identity

$$
\mathcal{L}\left(\zeta_{0} u\right)=\left(\mathcal{L} \zeta_{0}\right) u+\zeta_{0}(\mathcal{L} u)+2 \sum_{i, j=1}^{q} a_{i j}(t) X_{i} \zeta_{0} X_{j} u
$$

and the fact that the operators $\partial_{t}$ and $\widetilde{\Delta}_{h}$ commute, so that $\mathcal{L}$ and $\widetilde{\Delta}_{h}$ still commute.

Also Proposition 3.16 in [2] (Marchaud inequality on Carnot groups) still holds, with $L^{2}(\mathbb{G})$ norms replaced with $L^{2}\left(\mathbb{G}_{T}\right)$ norms, and this implies the following analog of Corollary 3.17 in [2].

Corollary 2.13. Let $u \in \mathcal{H}, u(0, \cdot)=0$, and assume that for $\varepsilon \in(0,1)$ and some integer $m>1$ the seminorm $|u|_{m, 1+\varepsilon}^{R}$ is finite. Then,

$$
|u|_{1}^{R} \leqslant c\left\{|u|_{m, 1+\varepsilon}^{R}+\|u\|_{L^{2}\left(\mathbb{G}_{T}\right)}\right\},
$$

with $c=c(\mathbb{G})$.

We are now in position to state the first step of our regularity estimate:

Proposition 2.14. Let $\zeta_{0}, \zeta \in C_{0}^{\infty}(\mathbb{G})$ with $\zeta_{0} \prec \zeta$. There exists $c=c\left(\zeta_{0}, \zeta, \mathbb{G}, v\right)>$ 0 such that

$$
\text { if } u \in \mathcal{H}_{0} \text { and } \mathcal{L} u \in L^{2}\left((0, T), W_{X^{R}, \text { loc }}^{s, 2}(\mathbb{G})\right) \text { then } u \in L^{2}\left((0, T), W_{X^{R}, \text { loc }}^{1,2}(\mathbb{G})\right)
$$

and

$$
\left\|\zeta_{0} u\right\|_{L^{2}\left((0, T), W_{X^{R}}^{1,2}(\mathbb{G})\right)} \leqslant c\left(\|\zeta \mathcal{L} u\|_{L^{2}\left((0, T), W_{X^{R}}^{s, 2}(\mathbb{G})\right)}+\|\zeta u\|_{L^{2}\left(\mathbb{G}_{T}\right)}\right) .
$$

Proof. Applying to $\zeta_{0} u$ Corollary 2.13 and Theorem 2.12 with $m=s+1$ and $\varepsilon=1 / s$, we can write:

$$
\left|\zeta_{0} u\right|_{1}^{R} \leqslant c\left\{\left|\zeta_{0} u\right|_{s+1,1+1 / s}^{R}+\left\|\zeta_{0} u\right\|_{L^{2}\left(\mathbb{G}_{T}\right)}\right\} \leqslant c\left(\sum_{j=0}^{s}|\zeta \mathcal{L} u|_{j}^{R}+\|\zeta u\|_{L^{2}\left(\mathbb{G}_{T}\right)}\right)
$$

From this inequality, by Propositions 2.9 and 2.8 we conclude the desired result. 
To iterate this result to higher-order derivatives, we first need a regularization result allowing to apply (2.11) to functions $u$ satisfying weaker assumptions.

Proposition 2.15. Let $u \in W^{1,2}\left((0, T), L_{\mathrm{loc}}^{2}(\mathbb{G})\right), u(0, \cdot)=0$, be a weak solution to $\mathcal{L} u=F \in L^{2}\left((0, T), L_{\mathrm{loc}}^{2}(\mathbb{G})\right)$ in the sense of Definition 2.2. If $F \in$ $L^{2}\left((0, T), W_{X^{R}, \text { loc }}^{s^{2}, 2}(\mathbb{G})\right)$ then $u \in L^{2}\left((0, T), W_{X^{R}, \text { loc }}^{1,2}(\mathbb{G})\right)$ and for every $\zeta, \zeta_{1} \in$ $C_{0}^{\infty}(\mathbb{G})$ with $\zeta \prec \zeta_{1}$ the following estimate holds:

$$
\|\zeta u\|_{L^{2}\left((0, T), W_{X^{R}}^{1,2}(\mathbb{G})\right)} \leqslant c\left\{\left\|\zeta_{1} F\right\|_{L^{2}\left((0, T), W_{X^{R}}^{s, 2}(\mathbb{G})\right)}+\left\|\zeta_{1} u\right\|_{L^{2}\left(\mathbb{G}_{T}\right)}\right\}
$$

with $c=c\left(\zeta_{0}, \zeta, \mathbb{G}, v\right)$.

Proof. Let us define the $\varepsilon$-mollified $u_{\varepsilon}$ of $u$ as follows. For $\phi \in C_{0}^{\infty}(\mathbb{G})$ such that

$$
\phi \geqslant 0, \phi(x)=0 \text { for }\|x\| \geqslant 1 \text { and } \int_{\mathbb{G}} \phi(x) \mathrm{d} x=1,
$$

define, for any $\varepsilon>0$,

$$
\phi_{\varepsilon}(x)=\varepsilon^{-Q} \phi\left(D_{\varepsilon^{-1}} x\right)
$$

and

$u_{\varepsilon}(t, x)=\left(\phi_{\varepsilon} * u\right)(t, x)=\int_{\mathbb{G}} \phi_{\varepsilon}(y) u\left(t, y^{-1} \circ x\right) \mathrm{d} y=\int_{\mathbb{G}} \phi_{\varepsilon}\left(x \circ z^{-1}\right) u(t, z) \mathrm{d} z$.

Now the function $u_{\varepsilon}$ is smooth with respect to $x$ (as can be seen computing $X_{I}^{R} u_{\varepsilon}$ ), while

$$
\frac{\partial u_{\varepsilon}}{\partial t}=\phi_{\varepsilon} * \frac{\partial u}{\partial t}
$$

and, for any couple of domains $K \Subset K^{\prime} \Subset \mathbb{G}$ and $\varepsilon$ small enough,

$$
\begin{aligned}
\left\|\frac{\partial u_{\varepsilon}}{\partial t}(t, \cdot)\right\|_{L^{2}(K)} & \leqslant\left\|\frac{\partial u}{\partial t}(t, \cdot)\right\|_{L^{2}\left(K^{\prime}\right)} \\
\left\|\frac{\partial u_{\varepsilon}}{\partial t}\right\|_{L^{2}\left((0, T), L^{2}(K)\right)} & \leqslant\left\|\frac{\partial u}{\partial t}\right\|_{L^{2}\left((0, T), L^{2}\left(K^{\prime}\right)\right)}
\end{aligned} .
$$

Here we have used Young's inequality in the form

$$
\left\|f * \phi_{\varepsilon}\right\|_{L^{2}(K)} \leqslant\|f\|_{L^{2}\left(K^{\prime}\right)}
$$

for $K \Subset K^{\prime}$, and $\varepsilon$ small enough, since $\phi_{\varepsilon}$ is compactly supported.

Also,

$$
u_{\varepsilon}(0, x)=\int_{\mathbb{G}} \phi_{\varepsilon}(y) u\left(0, y^{-1} \circ x\right) d y=0,
$$


hence $u_{\varepsilon} \in \mathcal{H}_{0}$ and we can apply to $u_{\varepsilon}$ the estimate proved in Proposition 2.14:

$$
\left\|\zeta u_{\varepsilon}\right\|_{L^{2}\left((0, T), W_{X^{R}}^{1,2}(\mathbb{G})\right)} \leqslant c\left\{\left\|\zeta_{1} \mathcal{L}\left(u_{\varepsilon}\right)\right\|_{L^{2}\left((0, T), W_{X^{R}}^{s, 2}(\mathbb{G})\right)}+\left\|\zeta_{1} u_{\varepsilon}\right\|_{L^{2}\left(\mathbb{G}_{T}\right)}\right\} .
$$

We claim that

$$
\mathcal{L}\left(u_{\varepsilon}\right)=F_{\varepsilon}
$$

for a.e. $t$ and a.e. $x$. This is not trivial since $\mathcal{L} u$ just exists in the above weak sense; hence, we cannot simply write $\mathcal{L}\left(u_{\varepsilon}\right)=(\mathcal{L} u)_{\varepsilon}$. However, for every $\varphi \in C_{0}^{\infty}(\mathbb{G})$, letting

$$
\mathcal{L}=-\partial_{t}+\mathcal{A}
$$

with

$$
\mathcal{A} u(t, x)=\sum_{i, j=1}^{q} a_{i j}(t) X_{i} X_{j} u(t, x)
$$

we can write:

$$
\int_{\mathbb{G}} \mathcal{L}\left(u_{\varepsilon}\right)(t, x) \varphi(x) \mathrm{d} x=\int_{\mathbb{G}}-\partial_{t}\left(u_{\varepsilon}\right)(t, x) \varphi(x) \mathrm{d} x+\int_{\mathbb{G}} u_{\varepsilon}(t, x) \mathcal{A} \varphi(x) \mathrm{d} x
$$

Next,

$$
\begin{aligned}
& \int_{\mathbb{G}} u_{\varepsilon}(t, x) \mathcal{A} \varphi(x) \mathrm{d} x \\
& =\int_{\mathbb{G}} \phi_{\varepsilon}(y)\left(\int \mathcal{A} \varphi(x) u\left(t, y^{-1} \circ x\right) \mathrm{d} x\right) \mathrm{d} y \\
& =\int_{\mathbb{G}} \phi_{\varepsilon}(y)\left(\int \mathcal{A} \varphi(y \circ z) u(t, z) \mathrm{d} z\right) \mathrm{d} y
\end{aligned}
$$

and

$$
\begin{aligned}
\int_{\mathbb{G}} \partial_{t}\left(u_{\varepsilon}\right)(t, x) \varphi(x) \mathrm{d} x & =\int_{\mathbb{G}}\left(\int \phi_{\varepsilon}(y) \partial_{t} u\left(t, y^{-1} \circ x\right) \mathrm{d} y\right) \varphi(x) \mathrm{d} x \\
& =\int_{\mathbb{G}} \phi_{\varepsilon}(y)\left(\int \partial_{t} u(t, z) \varphi(y \circ z) \mathrm{d} z\right) \mathrm{d} y
\end{aligned}
$$

letting $\psi_{y}(z)=\varphi(y \circ z)$

$$
\begin{aligned}
& \int_{\mathbb{G}} \mathcal{L}\left(u_{\varepsilon}\right)(t, x) \varphi(x) \mathrm{d} x \\
& \quad=\int_{\mathbb{G}} \phi_{\varepsilon}(y)\left(\int_{\mathbb{G}}-\partial_{t} u(t, z) \psi_{y}(z)+\mathcal{A} \psi_{y}(z) u(z) \mathrm{d} z\right) \mathrm{d} y
\end{aligned}
$$




$$
\begin{aligned}
& =\int_{\mathbb{G}} \phi_{\varepsilon}(y)\left(\int_{\mathbb{G}} \psi_{y}(z) F(t, z) \mathrm{d} z\right) \mathrm{d} y \\
& =\int_{\mathbb{G}} \varphi(x)\left(\int_{\mathbb{G}} \phi_{\varepsilon}(y) F\left(t, y^{-1} \circ x\right) \mathrm{d} y\right) \mathrm{d} x=\int_{\mathbb{G}} \varphi(x) F_{\varepsilon}(t, x) \mathrm{d} x
\end{aligned}
$$

and (2.15) follows. By known properties of the mollifiers, as $\varepsilon \rightarrow 0$ we have $\phi_{\varepsilon} * u \rightarrow u$ in $L^{2}\left(\mathbb{R}^{N}\right)$ as soon as $u \in L^{2}\left(\mathbb{R}^{N}\right)$. Also, for every left invariant differential operator $L$ we can write $L\left(\phi_{\varepsilon} * u\right)=\phi_{\varepsilon} * L u$ as soon as $L u$ exists in $L^{2}\left(\mathbb{R}^{N}\right)$. Therefore,

$$
\zeta_{1} \mathcal{L}\left(u_{\varepsilon}\right)=\zeta_{1} F_{\varepsilon} \rightarrow \zeta_{1} F \text { in } W_{X}^{k, 2}(\mathbb{G}) \text {, for a.e. } t
$$

as soon as $F \in L^{2}\left((0, T), W_{X, \text { loc }}^{k, 2}(\mathbb{G})\right)$.

To prove convergence in $L^{2}\left((0, T), W_{X^{R}, \text { loc }}^{s, 2}(\mathbb{G})\right)$ we make the following rough estimates (see Proposition 1.1):

$$
\begin{aligned}
\left\|\zeta_{1} \mathcal{L}\left(u_{\varepsilon}\right)-\zeta_{1} F\right\|_{L^{2}\left((0, T), W_{X}^{s, 2}(\mathbb{G})\right)} & \leqslant c\left\|\zeta_{1} F_{\varepsilon}-\zeta_{1} F\right\|_{L^{2}\left((0, T), W^{s, 2}(\mathbb{G})\right)} \\
& \leqslant c\left\|\zeta_{1} F_{\varepsilon}-\zeta_{1} F\right\|_{L^{2}\left((0, T), W_{X}^{s^{2}, 2}(\mathbb{G})\right)} .
\end{aligned}
$$

We want to show that, for $F \in L^{2}\left((0, T), W_{X, \text { loc }}^{s^{2}, 2}(\mathbb{G})\right)$,

$$
\left\|\zeta_{1} F_{\varepsilon}-\zeta_{1} F\right\|_{L^{2}\left((0, T), W_{X}^{s^{2}, 2}(\mathbb{G})\right)} \rightarrow 0
$$

Now:

$$
\begin{aligned}
& \left\|\zeta_{1} F_{\varepsilon}-\zeta_{1} F\right\|_{L^{2}\left((0, T), W_{X}^{s^{2}, 2}(\mathbb{G})\right)}^{2} \\
& \quad=\int_{0}^{T}\left\|\zeta_{1} F_{\varepsilon}(t, \cdot)-\zeta_{1} F(t, \cdot)\right\|_{W_{X}^{s^{2}, 2}(\mathbb{G})}^{2} \mathrm{~d} t \equiv \int_{0}^{T} g_{\varepsilon}(t) \mathrm{d} t
\end{aligned}
$$

where by (2.16), we already know that

$$
g_{\varepsilon}(t) \rightarrow 0 \text { for a.e. } t \in[0, T] \text {, as } \varepsilon \rightarrow 0 \text {. }
$$

To apply Lebesgue theorem and conclude the desired result we need to bound $g_{\varepsilon}$ with an integrable function independent of $\varepsilon$. Now:

$$
\begin{aligned}
\left\|\zeta_{1} F_{\varepsilon}(t, \cdot)-\zeta_{1} F(t, \cdot)\right\|_{W_{X}^{s^{2}, 2}(\mathbb{G})} & \leqslant\left\|\zeta_{1} F_{\varepsilon}(t, \cdot)\right\|_{W_{X}^{s^{2}, 2}(\mathbb{G})}+\left\|\zeta_{1} F(t, \cdot)\right\|_{W_{X}^{s^{2}, 2}(\mathbb{G})} \\
\left\|\zeta_{1} F_{\varepsilon}(t, \cdot)\right\|_{L^{2}(\mathbb{G})}^{2} & \leqslant\left\|F_{\varepsilon}(t, \cdot)\right\|_{L^{2}(K)}^{2} \leqslant\|F(t, \cdot)\|_{L^{2}\left(K^{\prime}\right)}^{2} \in L^{1}(0, T)
\end{aligned}
$$

where $K \Subset K^{\prime} \Subset \mathbb{G}$ and $\varepsilon$ small enough (see (2.13)). By (1.3), we have $X_{i}\left(F_{\varepsilon}\right)=$ $\left(X_{i} F\right)_{\varepsilon}$, then

$$
X_{i}\left(\zeta_{1} F_{\varepsilon}\right)=\left(X_{i} \zeta_{1}\right) F_{\varepsilon}+\zeta_{1}\left(X_{i} F\right)_{\varepsilon}
$$




$$
\begin{aligned}
\left\|X_{i}\left(\zeta_{1} F_{\varepsilon}(t, \cdot)\right)\right\|_{L^{2}(\mathbb{G})}^{2} & \leqslant c\left(\left\|F_{\varepsilon}(t, \cdot)\right\|_{L^{2}(K)}^{2}+\left\|\left(X_{i} F\right)_{\varepsilon}(t, \cdot)\right\|_{L^{2}(K)}^{2}\right) \\
& \leqslant c\left(\|F(t, \cdot)\|_{L^{2}\left(K^{\prime}\right)}^{2}+\left\|X_{i} F(t, \cdot)\right\|_{L^{2}\left(K^{\prime}\right)}^{2}\right) \in L^{1}(0, T),
\end{aligned}
$$

and an iterative reasoning allows to conclude (2.18). Recalling (2.17) and the fact that

$$
\left\|\zeta_{1} u_{\varepsilon}\right\|_{L^{2}\left(\mathbb{G}_{T}\right)} \rightarrow\left\|\zeta_{1} u\right\|_{L^{2}\left(\mathbb{G}_{T}\right)}
$$

we conclude that the right-hand side of (2.14) is bounded. Hence, the sequence $\zeta u_{\varepsilon}$ is bounded in $L^{2}\left((0, T), W_{X^{R}}^{1,2}(\mathbb{G})\right)$, and there exists a subsequence of $\zeta u_{\varepsilon}$ weakly converging in $L^{2}\left((0, T), W_{X^{R}}^{1,2}(\mathbb{G})\right)$ to some $g$ and in particular weakly converging in $L^{2}\left(\mathbb{G}_{T}\right)$ to $\zeta u$. This is enough to say that $\zeta u \in L^{2}\left((0, T), W_{X^{R}}^{1,2}(\mathbb{G})\right)$. Moreover,

$$
\begin{aligned}
\|\zeta u\|_{L^{2}\left((0, T), W_{X^{R}}^{1,2}(\mathbb{G})\right)} \leqslant \lim \inf \left\|\zeta u_{\varepsilon}\right\|_{L^{2}\left((0, T), W_{X^{R}}^{1,2}(\mathbb{G})\right)} \\
\leqslant c\left\{\left\|\zeta_{1} F\right\|_{L^{2}\left((0, T), W_{X^{R}}^{s, 2}(\mathbb{G})\right)}+\left\|\zeta_{1} u\right\|_{L^{2}\left(\mathbb{G}_{T}\right)}\right\}
\end{aligned}
$$

hence (2.12) holds.

We are now in position for the

Proof of Theorem 2.3. (i) We will prove (2.2) by induction on $k$. For $k=1$, this is exactly Proposition 2.15. Assume that (2.2) holds up to an integer $k$ and let $u \in$ $\mathcal{H}_{0}$ such that $\mathcal{L} u \in L^{2}\left((0, T), W_{X^{R}}^{k+s^{2}, 2}(\mathbb{G})\right)$. By the inductive assumption, $\zeta u \in$ $L^{2}\left((0, T), W_{X^{R}}^{k, 2}(\mathbb{G})\right)$. Let $X_{I}^{R}$ be a right invariant differential operator with $|I| \leqslant k$, then $\zeta X_{I}^{R} u \in L^{2}\left(\mathbb{G}_{T}\right)$. We would like to apply Proposition 2.15 to $X_{I}^{R} u$, but in order to do that we would need to know that $X_{I}^{R} u \in W^{1,2}\left((0, T), L_{\text {loc }}^{2}(\mathbb{G})\right)$ with $X_{I}^{R} u(0, \cdot)=0$, which is unclear. Then, let $u_{\varepsilon}$ be the mollified version of $u$ as in the proof of Proposition 2.15, so that:

$$
X_{I}^{R}\left(u_{\varepsilon}\right)(t, x)=\int_{\mathbb{G}}\left(X_{I}^{R} \phi_{\varepsilon}\right)\left(x \circ z^{-1}\right) u(t, z) \mathrm{d} z
$$

which is a smooth function in $x$, and since $X_{I}^{R} \phi_{\varepsilon}$ is integrable (although its $L^{1}(\mathbb{G})$ norm is not uniformly bounded with respect to $\varepsilon$ ) we have

$$
X_{I}^{R}\left(u_{\varepsilon}\right) \in L^{2}\left((0, T), L_{\text {loc }}^{2}(\mathbb{G})\right)
$$

(see (2.13)) and since $\partial_{t} u \in L^{2}\left((0, T), L_{\text {loc }}^{2}(\mathbb{G})\right)$, the same is true for $\partial_{t} X_{I}^{R}\left(u_{\varepsilon}\right)$, which equals $X_{I}^{R}\left(\partial_{t} u\right)_{\varepsilon}$. Then,

$$
X_{I}^{R}\left(u_{\varepsilon}\right) \in W^{1,2}\left((0, T), L_{\mathrm{loc}}^{2}(\mathbb{G})\right)
$$


which also implies

$$
X_{I}^{R}\left(u_{\varepsilon}\right)(0, x)=\int_{\mathbb{G}}\left(X_{I}^{R} \phi_{\varepsilon}\right)\left(x \circ z^{-1}\right) u(0, z) \mathrm{d} z=0
$$

since $u(0, \cdot)=0$ in $L^{2}(\mathbb{G})$. We claim that

$$
\mathcal{L}\left(X_{I}^{R}\left(u_{\varepsilon}\right)\right)=X_{I}^{R}\left(\mathcal{L}\left(u_{\varepsilon}\right)\right)=X_{I}^{R}\left(F_{\varepsilon}\right)
$$

at least in weak sense. Actually, noting that $\mathcal{L}$ and $X_{I}^{R}$ commute,

$$
\begin{aligned}
\int_{\mathbb{G}} \mathcal{L}\left(X_{I}^{R}\left(u_{\varepsilon}\right)\right)(t, x) \varphi(x) \mathrm{d} x & =\int_{\mathbb{G}} X_{I}^{R}\left(\mathcal{L}\left(u_{\varepsilon}\right)\right)(t, x) \varphi(x) \mathrm{d} x \\
& =-\int_{\mathbb{G}} \mathcal{L}\left(u_{\varepsilon}\right)(t, x)\left(X_{I}^{R} \varphi\right)(x) \mathrm{d} x
\end{aligned}
$$

since $X_{I}^{R} \varphi \in C_{0}^{\infty}(\mathbb{G})$ and $\mathcal{L}\left(u_{\varepsilon}\right)=F_{\varepsilon}$ for a.e. $t$ and $x($ see $(2.15))$

$$
=-\int_{\mathbb{G}} F_{\varepsilon}(t, x)\left(X_{I}^{R} \varphi\right)(x) \mathrm{d} x=\int_{\mathbb{G}} X_{I}^{R}\left(F_{\varepsilon}\right)(t, x) \varphi(x) \mathrm{d} x
$$

for a.e. $t$. Therefore, we can apply Proposition 2.15 to $X_{I}^{R}\left(u_{\varepsilon}\right)$ getting

$$
\begin{aligned}
& \left\|\zeta X_{I}^{R}\left(u_{\varepsilon}\right)\right\|_{L^{2}\left((0, T), W_{X}^{1,2}(\mathbb{G})\right)} \\
& \quad \leqslant c\left\{\left\|\zeta_{1} X_{I}^{R}\left(F_{\varepsilon}\right)\right\|_{L^{2}\left((0, T), W_{X}^{s, 2}(\mathbb{G})\right)}+\left\|\zeta_{1} X_{I}^{R}\left(u_{\varepsilon}\right)\right\|_{L^{2}\left(\mathbb{G}_{T}\right)}\right\} .
\end{aligned}
$$

Noting that

$$
\left\|\zeta_{1} X_{I}^{R}\left(u_{\varepsilon}\right)\right\|_{L^{2}\left(\mathbb{G}_{T}\right)} \leqslant\left\|\zeta_{1} X_{I^{\prime}}^{R}\left(u_{\varepsilon}\right)\right\|_{L^{2}\left((0, T), W_{X^{R}}^{1,2}(\mathbb{G})\right)}
$$

for some $I^{\prime}$ with $\left|I^{\prime}\right|=|I|-1$, we can proceed iteratively getting, for some different cutoff function $\zeta_{2} \succ \zeta_{1}$,

$$
\left\|\zeta X_{I}^{R}\left(u_{\varepsilon}\right)\right\|_{L^{2}\left((0, T), W_{X^{R}}^{1,2}(\mathbb{G})\right)} \leqslant c\left\{\left\|\zeta_{2} X_{I}^{R}\left(F_{\varepsilon}\right)\right\|_{L^{2}\left((0, T), W_{X}^{s, 2}(\mathbb{G})\right)}+\left\|\zeta_{2} u_{\varepsilon}\right\|_{L^{2}\left(\mathbb{G}_{T}\right)}\right\} .
$$

From this bound, which is uniform with respect to $\varepsilon$, reasoning like in the proof of Proposition 2.15 we read that, under the assumption $X_{I}^{R} F \in L^{2}\left((0, T), W_{X^{R}}^{s^{2}, 2}(\mathbb{G})\right)$, which is true as soon as $F \in L^{2}\left((0, T), W_{X^{R}}^{k+s^{2}, 2}(\mathbb{G})\right)$, we have the uniform boundedness of

$$
\left\|\zeta X_{I}^{R}\left(u_{\varepsilon}\right)\right\|_{L^{2}\left((0, T), W_{X^{R}}^{1,2}(\mathbb{G})\right)},
$$


which implies the weak convergence in $L^{2}\left((0, T), W_{X^{R}}^{1,2}(\mathbb{G})\right)$ of (a subsequence of) $\zeta X_{I}^{R}\left(u_{\varepsilon}\right)$ to some $g$. In particular, the convergence is in $L^{2}\left(\mathbb{G}_{T}\right)$, which implies that for every $\eta \in L^{2}(0, T)$ and $\phi \in C_{0}^{\infty}(\mathbb{G})$

$$
\int_{0}^{T} \eta(t) \int_{\mathbb{G}} \zeta(x) X_{I}^{R}\left(u_{\varepsilon}\right)(t, x) \phi(x) \mathrm{d} x \mathrm{~d} t \rightarrow \int_{0}^{T} \eta(t) \int_{\mathbb{G}} g(t, x) \phi(x) \mathrm{d} x \mathrm{~d} t .
$$

Pick the cutoff function $\zeta(x)=1$ on some bounded open set $\Omega$, then for every $\phi \in C_{0}^{\infty}(\Omega)$ we have

$$
\int_{0}^{T} \eta(t) \int_{\mathbb{G}} X_{I}^{R}\left(u_{\varepsilon}\right)(t, x) \phi(x) \mathrm{d} x \mathrm{~d} t \rightarrow \int_{0}^{T} \eta(t) \int_{\mathbb{G}} g(t, x) \phi(x) \mathrm{d} x \mathrm{~d} t .
$$

On the other hand,

$$
\begin{aligned}
& \int_{0}^{T} \eta(t) \int_{\mathbb{G}} X_{I}^{R}\left(u_{\varepsilon}\right)(t, x) \phi(x) \mathrm{d} x \mathrm{~d} t \\
& \quad=(-1)^{|I|} \int_{0}^{T} \eta(t) \int_{\mathbb{G}} u_{\varepsilon}(t, x) X_{I}^{R} \phi(x) \mathrm{d} x \mathrm{~d} t \\
& \quad \rightarrow(-1)^{|I|} \int_{0}^{T} \eta(t) \int_{\mathbb{G}} u(t, x) X_{I}^{R} \phi(x) \mathrm{d} x \mathrm{~d} t,
\end{aligned}
$$

hence

$$
\int_{0}^{T} \eta(t) \int_{\mathbb{G}} g(t, x) \phi(x) \mathrm{d} x \mathrm{~d} t=(-1)^{|I|} \int_{0}^{T} \eta(t) \int_{\mathbb{G}} u(t, x) X_{I}^{R} \phi(x) \mathrm{d} x \mathrm{~d} t
$$

which implies, for a.e. $t$ and a.e. $x \in \Omega$,

$$
g(t, x)=X_{I}^{R} u(t, x)
$$

in the sense of weak derivatives. This means that $\zeta X_{I}^{R} u \in L^{2}\left((0, T), W_{X^{R}}^{1,2}(\mathbb{G})\right)$ and $\zeta X_{I}^{R}\left(u_{\varepsilon}\right) \rightarrow \zeta X_{I}^{R} u$ weakly in $L^{2}\left((0, T), W_{X^{R}}^{1,2}(\mathbb{G})\right)$, which also implies, by (2.19),

$$
\left\|\zeta X_{I}^{R} u\right\|_{L^{2}\left((0, T), W_{X^{R}}^{1,2}(\mathbb{G})\right)} \leqslant c\left\{\left\|\zeta_{2} X_{I}^{R} F\right\|_{L^{2}\left((0, T), W_{X^{R}}^{s, 2}(\mathbb{G})\right)}+\left\|\zeta_{2} u\right\|_{L^{2}\left(\mathbb{G}_{T}\right)}\right\} .
$$

So we are done.

(ii) Let $\zeta \in C_{0}^{\infty}(\mathbb{G})$ and $u \in W_{X^{R}, \text { loc }}^{2 s, 2}(\mathbb{G})$. Inequalities

$$
\|\zeta u\|_{W_{X}^{2,2}(\mathbb{G})} \leqslant c\|\zeta u\|_{W^{2,2}(\mathbb{G})} \leqslant c\|\zeta u\|_{W_{X^{R}}^{2 s, 2}(\mathbb{G})}
$$

show that

$$
W_{X^{R}, \mathrm{loc}}^{2 s, 2}(\mathbb{G}) \subset W_{X, \mathrm{loc}}^{2,2}(\mathbb{G}) .
$$


Let $u \in W^{1,2}\left((0, T), L_{\text {loc }}^{2}(\mathbb{G})\right), u(0, \cdot)=0$, be a weak solution to $\mathcal{L} u=F \in$ $L^{2}\left((0, T), W_{X^{R}, \text { loc }}^{h, 2}(\mathbb{G})\right)$. By point (i), if $\zeta_{1} F \in L^{2}\left((0, T), W_{X^{R}}^{k+s^{2}-1,2}(\mathbb{G})\right)$, then $\zeta u \in L^{2}\left((0, T), W_{X^{R}}^{k, 2}(\mathbb{G})\right)$. In particular, if $h \geq 2 s+s^{2}-1$ then $u \in L^{2}\left((0, T), W_{X, \text { loc }}^{2,2}(\mathbb{G})\right)$ and this implies that $u$ is actually a strong solution to the equation $\mathcal{L} u=F$, so that for a.e. $t$ and a.e. $x$ we have

$$
-u_{t}(t, x)+\sum_{i, j=1}^{q} a_{i j}(t) X_{i} X_{j} u(t, x)=F(t, x) .
$$

This identity allows to transfer further $x$-regularity of both $F$ and $u$ to $u_{t}$ : if, for some $k=1,2,3, \ldots$, we know that $h \geq k+2 s+s^{2}-1$, then by point (i) $u \in L^{2}\left((0, T), W_{X^{R}, \text { loc }}^{k+2 s, 2}(\mathbb{G})\right)$, so that $X_{i} X_{j} u \in L^{2}\left((0, T), W_{X^{R}, \text { loc }}^{k, 2}(\mathbb{G})\right)$, hence by (2.20) $u_{t} \in L^{2}\left((0, T), W_{X^{R}, \text { loc }}^{k, 2}(\mathbb{G})\right)$ and $u \in W^{1,2}\left((0, T), W_{X^{R}, \text { loc }}^{k, 2}(\mathbb{G})\right)$.

This implies that for $|I| \leqslant k, X_{I}^{R} u \in C^{0}\left([0, T], L_{\text {loc }}^{2}(\mathbb{G})\right)$. Moreover, we can write, for every $t_{1}, t_{2} \in[0, T]$ and a.e. $x \in \mathbb{G}$,

$$
\begin{gathered}
u\left(t_{2}, x\right)-u\left(t_{1}, x\right)=\int_{t_{1}}^{t_{2}} \partial_{t} u(t, x) d t \\
X_{I}^{R} u\left(t_{2}, x\right)-X_{I}^{R} u\left(t_{1}, x\right)=\int_{t_{1}}^{t_{2}} \partial_{t} X_{I}^{R} u(t, x) \mathrm{d} t .
\end{gathered}
$$

Letting $t_{1}=0$ in (2.21), we get

$$
u\left(t_{2}, x\right)=\int_{0}^{t_{2}} \partial_{t} u(t, x) \mathrm{d} t
$$

an identity which can also be differentiated with respect to $X_{I}^{R}$, giving

$$
X_{I}^{R} u\left(t_{2}, x\right)=\int_{0}^{t_{2}} X_{I}^{R} \partial_{t} u(t, x) \mathrm{d} t,
$$

which implies

$$
X_{I}^{R} u(0, \cdot)=0
$$

This completes the proof of (ii).

(iii) Next, multiplying both sides of (2.22) for $\zeta \in C_{0}^{\infty}(\mathbb{G})$ and taking $L^{2}(\mathbb{G})$-norms we get, recalling that $X_{I}^{R}$ commutes with $\mathcal{L}$ :

$$
\begin{aligned}
& \int_{\mathbb{G}} \zeta(x)^{2}\left|X_{I}^{R} u\left(t_{2}, x\right)-X_{I}^{R} u\left(t_{2}, x\right)\right|^{2} \mathrm{~d} x \\
& \quad \leqslant \int_{\mathbb{G}} \zeta(x)^{2}\left|\int_{t_{1}}^{t_{2}}\left\{-X_{I}^{R} \mathcal{L} u(t, x)+\sum_{i, j=1}^{q} a_{i j}(t) X_{i} X_{j} X_{I}^{R} u(t, x)\right\} \mathrm{d} t\right|^{2} \mathrm{~d} x
\end{aligned}
$$




$$
\begin{aligned}
& \leqslant \int_{\mathbb{G}} \zeta(x)^{2}\left(\int_{t_{1}}^{t_{2}}\left\{\left|X_{I}^{R} F(t, x)\right|+c_{v} \sum_{i, j=1}^{q}\left|X_{i} X_{j} X_{I}^{R} u(t, x)\right|\right\} \mathrm{d} t\right)^{2} \mathrm{~d} x \\
& \leqslant \int_{\mathbb{G}} \zeta(x)^{2}\left|t_{2}-t_{1}\right|\left\{\int_{0}^{T}\left|X_{I}^{R} F(t, x)\right|^{2} \mathrm{~d} t+c_{v} \sum_{i, j=1}^{q} \int_{0}^{T}\left|X_{i} X_{j} X_{I}^{R} u(t, x)\right|^{2} \mathrm{~d} t\right\} \mathrm{d} x \\
& =\left|t_{2}-t_{1}\right|\left\{\left\|\zeta X_{I}^{R} F\right\|_{L^{2}\left(\mathbb{G}_{T}\right)}^{2}+c_{v} \sum_{i, j=1}^{q}\left\|\zeta X_{i} X_{j} X_{I}^{R} u\right\|_{L^{2}\left(\mathbb{G}_{T}\right)}^{2}\right\} .
\end{aligned}
$$

By point (i) of this theorem, this implies that

$$
\begin{aligned}
& \sup _{0<t_{1}<t_{2}<T} \frac{\int_{\mathbb{G}} \zeta(x)^{2}\left|X_{I}^{R} u\left(t_{2}, x\right)-X_{I}^{R} u\left(t_{2}, x\right)\right|^{2} d x}{\left|t_{2}-t_{1}\right|} \\
& \leqslant c\left\{\left\|\zeta_{1} F\right\|_{L^{2}\left((0, T), W_{X^{R}}^{h, 2}(\mathbb{G})\right)}+\left\|\zeta_{1} u\right\|_{L^{2}\left(\mathbb{G}_{T}\right)}\right\}^{2}
\end{aligned}
$$

for some $h$ large enough and any cutoff function $\zeta_{1}$ such that $\zeta \prec \zeta_{1}$. On the other hand, letting

$$
v(x)=u\left(t_{2}, x\right)-u\left(t_{2}, x\right)
$$

and noting that every cartesian derivative $\partial_{x}^{\alpha} v(x)$ can be bounded, uniformly on a compact set of $\mathbb{G}$ by a suitable linear combination of $X_{I}^{R} v$, we arrive to a bound

$$
\begin{aligned}
& \sup _{0<t_{1}<t_{2}<T} \frac{\left\|\zeta\left[\partial_{x}^{\alpha} u\left(t_{2}, \cdot\right)-\partial_{x}^{\alpha} u\left(t_{1}, \cdot\right)\right]\right\|_{L^{2}(\mathbb{G})}}{\left|t_{2}-t_{1}\right|^{1 / 2}} \\
& \quad \leqslant c\left\{\left\|\zeta_{1} F\right\|_{L^{2}\left((0, T), W_{X}^{h_{1}, 2}(\mathbb{G})\right)}+\left\|\zeta_{1} u\right\|_{L^{2}\left(\mathbb{G}_{T}\right)}\right\}
\end{aligned}
$$

for some integer $h_{1}>h$. And since also the sup of $\left|\partial_{x}^{\alpha} u\left(t_{2}, \cdot\right)-\partial_{x}^{\alpha} u\left(t_{1}, \cdot\right)\right|$ can be bounded, by Sobolev embeddings, by suitable $L^{2}$ norms of higher order derivatives, we also have a control

$$
\begin{aligned}
& \sup _{0<t_{1}<t_{2}<T} \sup _{x \in \mathbb{G}} \frac{\left|\zeta(x)\left[\partial_{x}^{\alpha} u\left(t_{2}, x\right)-\partial_{x}^{\alpha} u\left(t_{1}, x\right)\right]\right|}{\left|t_{2}-t_{1}\right|^{1 / 2}} \\
& \leqslant c\left\{\left\|\zeta_{1} F\right\|_{L^{2}\left((0, T), W_{X^{R}}^{h_{2}, 2}(\mathbb{G})\right)}+\left\|\zeta_{1} u\right\|_{L^{2}\left(\mathbb{G}_{T}\right)}\right\}
\end{aligned}
$$

for some integer $h_{2}>h_{1}$. Also, since $\partial_{x}^{\alpha} u(0, x)=0$, this implies

$$
\sup _{x \in \mathbb{G}}\left|\zeta(x) \partial_{x}^{\alpha} u(t, x)\right| \leqslant c|t|^{1 / 2}\left\{\left\|\zeta_{1} F\right\|_{L^{2}\left((0, T), W_{X^{R}}^{h_{2}, 2}(\mathbb{G})\right)}+\left\|\zeta_{1} u\right\|_{L^{2}\left(\mathbb{G}_{T}\right)}\right\} .
$$

This ends the proof of (iii). The previous result also shows that

$$
\zeta_{1} F \in L^{2}(0, T), C^{\infty}(\mathbb{G}) \Longrightarrow \zeta u \in C^{0}\left([0, T], C^{\infty}(\mathbb{G})\right)
$$


Then, the equality

$$
u_{t}=\sum_{i, j=1}^{q} a_{i j}(t) X_{i} X_{j} u-F
$$

also implies that

$$
\zeta u_{t} \in L^{2}\left((0, T), C^{\infty}(\mathbb{G})\right),
$$

that is (iv).

We end this section with an easy example showing that the regularity properties of the solution cannot be improved for bounded measurable coefficients $a_{i j}(t)$.

Example 2.16. Let us consider the uniformly parabolic operator

$$
\mathcal{L} u=-u_{t}+a(t) u_{x x}
$$

with $a \in L^{\infty}(\mathbb{R}), a(t) \geq v>0$. The function

$$
u(t, x)=\exp \left(-\int_{0}^{t} a(\tau) \mathrm{d} \tau\right) \sin x
$$

satisfies $\mathcal{L} u=0 ; u$ is smooth w.r.t. $x$ and only Lipschitz continuous w.r.t. $t$. Let

$$
U(t, x)=t^{\alpha} u(t, x) \text { for some } \alpha \in\left(\frac{1}{2}, 1\right) .
$$

Then, $U$ solves the problem

$$
\left\{\begin{array}{l}
\mathcal{L} U=F \text { for } x \in \mathbb{R}, t>0 \\
U(0, x)=0
\end{array}\right.
$$

with $F(t, x)=-\alpha t^{\alpha-1} u(t, x)$, so that, as soon as $\alpha>\frac{1}{2}$,

$$
F \in L^{2}((0, T) \times \mathbb{R}) .
$$

Moreover,

$$
U_{t}(t, x)=\alpha t^{\alpha-1} u(t, x)-t^{\alpha} a(t) u(t, x) \in L^{2}\left((0, T), C^{\infty}(\mathbb{R})\right)
$$

Hence,

$$
U \in W^{1,2}\left((0, T), C^{\infty}(\mathbb{R})\right) \cap C^{0, \alpha}\left([0, T], C^{\infty}(\mathbb{R})\right) .
$$

Since $\alpha>\frac{1}{2}$ can be chosen as close to $1 / 2$ as we want, this shows that the regularity with respect to $t$ expressed by Theorem 2.3 cannot be improved. Also, note that the Hölder continuity w.r.t. $t$ cannot be improved to Lipschitz continuity just remaining far off $t=0$ : if we multiply the above $U(t, x)$ for $\left|t-t_{0}\right|^{\alpha}$ we get a similar example exhibiting a $\alpha$-Hölder continuity w.r.t. $t$ near $t=t_{0}$. 


\section{Regularization of distributional solutions}

In this section, we want to extend our smoothness result, established in Theorem 2.3 (iv) for functions in $W^{1,2}\left((0, T), L_{\text {loc }}^{2}(\mathbb{G})\right)$, to more general distributions. First of all, we need to make precise the distributional notions that we will use.

Definition 3.1. Let $\Omega \subseteq \mathbb{G}$ be an open set. We will say that $u \in L^{2}\left((0, T), \mathcal{D}^{\prime}(\Omega)\right)$ if $u \in \mathcal{D}^{\prime}\left(\Omega_{T}\right)$ and for every $\phi \in \mathcal{D}(\Omega)$ there exists a function $h_{\phi} \in L^{2}(0, T)$ such that for every $\psi \in \mathcal{D}(0, T)$,

$$
\langle u, \phi \otimes \psi\rangle=\int_{0}^{T} h_{\phi}(t) \psi(t) \mathrm{d} t .
$$

In this case we will write, more transparently, $h_{\phi}(t)=\langle u(t, \cdot), \phi\rangle$ and

$$
\langle u, \phi(x) \psi(t)\rangle=\int_{0}^{T}\langle u(t, \cdot), \phi\rangle \psi(t) \mathrm{d} t
$$

for every $\phi \in \mathcal{D}(\Omega)$ and $\psi \in \mathcal{D}(0, T)$ (and therefore also for every $\psi \in L^{2}(0, T)$ ).

Analogously, we will say that $u \in W^{1,2}\left((0, T), \mathcal{D}^{\prime}(\Omega)\right)$ if $u \in \mathcal{D}^{\prime}\left(\Omega_{T}\right)$ with both $u$ and its distributional derivative $\partial_{t} u$ belonging to $L^{2}\left((0, T), \mathcal{D}^{\prime}(\Omega)\right)$.

We will say that $u$ is a distributional solution to $\mathcal{L} u=F$ in $\Omega_{T}$, with $F \in$ $L^{2}\left((0, T), \mathcal{D}^{\prime}(\Omega)\right)$ if $u \in W^{1,2}\left((0, T), \mathcal{D}^{\prime}(\Omega)\right)$ and:

$$
\left\langle-\partial_{t} u(t, \cdot), \phi\right\rangle+\sum_{i, j=1}^{q} a_{i j}(t)\left\langle X_{i} X_{j} u(t, \cdot), \phi\right\rangle=\langle F(t, \cdot), \phi\rangle
$$

for every $\phi \in \mathcal{D}(\Omega)$ and a.e. $t \in(0, T)$, or equivalently:

$$
\begin{aligned}
& \int_{0}^{T}\left\{\left\langle-\partial_{t} u(t, \cdot), \phi\right\rangle+\sum_{i, j=1}^{q} a_{i j}(t)\left\langle u(t, \cdot), X_{i} X_{j} \phi\right\rangle\right\} \psi(t) \mathrm{d} t \\
& =\int_{0}^{T}\langle F(t, \cdot), \phi\rangle \psi(t) \mathrm{d} t
\end{aligned}
$$

$\forall \phi \in \mathcal{D}(\Omega), \psi \in L^{2}(0, T)$

The proof of a regularity result for distributional solutions usually begins identifying the given distribution, locally, with some derivative of a continuous function, in view of the classical result about the local structure of distributions. For distributions in the class $L^{2}\left((0, T), \mathcal{D}^{\prime}(\Omega)\right)$, we could not find in the literature any reference for a similar result. So we will explicitly assume that our distribution could be seen, on a fixed domain compactly contained in $\Omega$, as a space derivative of a suitable function:

Definition 3.2. Let $u \in L^{2}\left((0, T), \mathcal{D}^{\prime}(\Omega)\right)$ for some open set $\Omega \subseteq \mathbb{G}$. We will say that $u$ satisfies the $x$-finite order assumption on $\Omega$ if: 
there exists a function $h \in L^{2}\left((0, T), L_{\text {loc }}^{1}(\Omega)\right)$ and a multiindex $\alpha$ such that

$$
u=\frac{\partial^{\alpha} h}{\partial x^{\alpha}} \text { in } \mathcal{D}^{\prime}\left(\Omega_{T}\right)
$$

that is

$$
\langle u, \phi(x) \psi(t)\rangle=\int_{0}^{T}\left((-1)^{|\alpha|} \int_{\Omega^{\prime}} h(t, x) \frac{\partial^{\alpha} \phi}{\partial x^{\alpha}}(x) \mathrm{d} x\right) \psi(t) \mathrm{d} t
$$

$\forall \phi \in \mathcal{D}(\Omega), \psi \in L^{2}(0, T)$.

If $u \in W^{1,2}\left((0, T), \mathcal{D}^{\prime}(\Omega)\right)$, we will say that $u$ satisfies the $x$-finite order assumption on $\Omega$ if (3.1) holds with $h \in W^{1,2}\left((0, T), L_{\text {loc }}^{1}(\Omega)\right)$.

Note that if $u \in W^{1,2}\left((0, T), \mathcal{D}^{\prime}(\Omega)\right)$ satisfies the $x$-finite order assumption on $\Omega^{\prime}$, then $h \in C^{0}\left([0, T], L_{\text {loc }}^{1}(\Omega)\right)$. In particular, saying that $u(0, \cdot)=0$ means that $h(0, \cdot)=0$ a.e. in $\Omega$.

The aim of this section is to prove that:

Theorem 3.3. For some bounded domain $\Omega \subset \mathbb{G}$, let $u$ be a distributional solution to $\mathcal{L} u=F$ in $\Omega_{T}$ with $F \in L^{2}\left((0, T), \mathcal{D}^{\prime}(\Omega)\right)$. Assume that $u$ satisfies the $x$-finite order assumption (see Definition 3.2) and $u(0, \cdot)=0$ in $\Omega$. Then, for every domain $\Omega^{\prime} \Subset \Omega$, if

$$
F \in L^{2}\left((0, T), C^{\infty}(\bar{\Omega})\right)
$$

then

$$
u \in C^{0}\left([0, T], C^{\infty}\left(\overline{\Omega^{\prime}}\right)\right) \text { and } u_{t} \in L^{2}\left((0, T), C^{\infty}\left(\overline{\Omega^{\prime}}\right)\right) .
$$

In order to prove Theorem 3.3, we will follow the technique used in [2, §4] for sublaplacians. Therefore, some proofs, which contain almost straightforward adaptations of corresponding arguments in [2, §4], will be omitted.

Let us consider the second-order differential operator

$$
\mathcal{L}^{R}=\sum_{j=1}^{N}\left(X_{j}^{R}\right)^{2}
$$

built using the whole canonical base of right invariant vector fields. This is a right invariant (but no longer homogeneous) uniformly elliptic operator in $\mathbb{G}$, which at the origin coincides with the standard Laplacian. Therefore, the fundamental solution of the Laplacian, $\gamma(x)=c_{N}|x|^{2-N}$, can be used to build a parametrix for $\mathcal{L}^{R}$ : letting $\tilde{\gamma}(x)=\gamma(x) \eta(x)$, where $\eta$ is a cutoff function equal to 1 in a neighborhood of the origin, one can prove the following:

Proposition 3.4. (see [2, Prop. 4.2.]) Let $V \subset \mathbb{G}$ be a neighborhood of the origin. There exist $\tilde{\gamma} \in C^{\infty}(\mathbb{G} \backslash\{0\})$ and $\omega \in C^{\infty}(\mathbb{G} \backslash\{0\})$, both supported in $V$, satisfying

$$
|\tilde{\gamma}(x)| \leqslant \frac{c}{|x|^{N-2}}
$$




$$
\begin{aligned}
\left|\partial_{x_{i}} \tilde{\gamma}(x)\right| & \leqslant \frac{c}{|x|^{N-1}} \quad i=1,2, \ldots, N \\
|\omega(x)| & \leqslant \frac{c}{|x|^{N-2}}
\end{aligned}
$$

and such that in the sense of distributions

$$
\mathcal{L}^{R} \tilde{\gamma}=-\delta+\omega .
$$

(Here $\delta$ is the Dirac mass as a distribution in $\mathbb{R}^{N}$.)

Let us now consider three open sets in $\mathbb{G}, \Omega^{\prime} \Subset \Omega^{\prime \prime} \Subset \Omega$ and let $V$ be a neighborhood of the origin such that $V^{-1} \circ \Omega^{\prime} \subset \Omega^{\prime \prime}$. Define $\tilde{\gamma}$ as in Proposition 3.4, with $\tilde{\gamma}$ supported in $V$. The convolution with $\tilde{\gamma}$ defines a regularizing operator that acts on functions $u \in L_{\text {loc }}^{1}\left(\mathbb{G}_{T}\right)$ as follows. For every $x \in \Omega^{\prime}$ and $t \in[0, T]$, we set

$$
T_{V} u(t, x)=(\tilde{\gamma} * u(t, \cdot))(x)=\int_{\mathbb{G}} \tilde{\gamma}\left(x \circ y^{-1}\right) u(t, y) \mathrm{d} y .
$$

The subscript $V$ in $T_{V}$ recalls that the definition of the operator depends on the choice of the neighborhood $V$ used to define $\tilde{\gamma}$.

Note that

$$
T_{V}: L^{2}\left((0, T), L^{1}\left(\Omega^{\prime \prime}\right)\right) \longrightarrow L^{2}\left((0, T), L^{1}\left(\Omega^{\prime}\right)\right) .
$$

Namely, for $x \in \Omega^{\prime}$ and $x \circ y^{-1} \in \operatorname{sprt} \tilde{\gamma}$, the point $y=\left(x \circ y^{-1}\right)^{-1} \circ x$ ranges in $V^{-1} \circ \Omega^{\prime} \subset \Omega^{\prime \prime}$; hence, introducing characteristic functions,

$$
\chi_{\Omega^{\prime}}(x) T_{V} u(t, x)=\int_{\mathbb{G}}\left(\tilde{\gamma} \chi_{V}\right)\left(x \circ y^{-1}\right) u(t, y) \chi_{\Omega^{\prime \prime}}(y) \mathrm{d} y,
$$

or

$$
\chi_{\Omega^{\prime}} T_{V} u(t, \cdot)=\tilde{\gamma} \chi_{V} * u(t, \cdot) \chi_{\Omega^{\prime \prime}}
$$

which by Young's inequality gives, at least for a.e. $t$,

$$
\left\|T_{V} u(t, \cdot)\right\|_{L^{1}\left(\Omega^{\prime}\right)} \leqslant\|\widetilde{\gamma}\|_{L^{1}(V)}\|u(t, \cdot)\|_{L^{1}\left(\Omega^{\prime \prime}\right)}
$$

and hence

$$
\left\|T_{V} u\right\|_{L^{2}\left((0, T), L^{1}\left(\Omega^{\prime}\right)\right)} \leqslant\|\tilde{\gamma}\|_{L^{1}(V)}\|u\|_{L^{2}\left((0, T), L^{1}\left(\Omega^{\prime \prime}\right)\right)} .
$$

Also, $T_{V}$ acts on distributions $u \in L^{2}\left((0, T), \mathcal{D}^{\prime}(\Omega)\right)$ as follows. For every $\varphi \in$ $\mathcal{D}\left(\Omega^{\prime}\right)$ we set

$$
\left\langle T_{V} u(t, \cdot), \varphi\right\rangle=\left\langle u(t, \cdot), T_{V}^{*} \varphi\right\rangle
$$


where

$$
T_{V}^{*} \varphi(y)=\int_{\mathbb{G}} \tilde{\gamma}\left(x \circ y^{-1}\right) \varphi(x) \mathrm{d} x .
$$

Observe that the assumption on $V$ implies that $T_{V}^{*} \varphi$ is a test function in $\Omega^{\prime}$. Namely, for $x \in \operatorname{sprt} \varphi$ and $x \circ y^{-1} \in \operatorname{sprt} \tilde{\gamma}$ the point $y$ ranges in $\Omega^{\prime \prime} \Subset \Omega$. The function $T_{V}^{*} \varphi$ is smooth, as one can see writing

$$
T_{V}^{*} \varphi(y)=\int_{\mathbb{G}} \tilde{\gamma}(z) \varphi(z \circ y) \mathrm{d} x
$$

and computing left invariant derivatives

$$
X_{I}\left(T_{V}^{*} \varphi\right)(y)=\int_{\mathbb{G}} \tilde{\gamma}(z)\left(X_{I} \varphi\right)(z \circ y) \mathrm{d} x .
$$

Therefore, the pairing (3.6) is well defined. Also, from the previous identity we easily read that if $\varphi_{k} \rightarrow 0$ in $\mathcal{D}(\Omega)$ then $T_{V}^{*} \varphi_{k} \rightarrow 0$ in $\mathcal{D}\left(\Omega^{\prime}\right)$. Hence, $T_{V} u(t, \cdot) \in \mathcal{D}^{\prime}\left(\Omega^{\prime}\right)$. Moreover,

$$
\int_{0}^{T}\left|\left\langle T_{V} u(t, \cdot), \varphi\right\rangle\right|^{2} \mathrm{~d} t=\int_{0}^{T}\left|\left\langle u(t, \cdot), T_{V}^{*} \varphi\right\rangle\right|^{2} \mathrm{~d} t<\infty
$$

(just by definition of $L^{2}\left((0, T), \mathcal{D}^{\prime}(\Omega)\right)$ ), so that

$$
T_{V}: L^{2}\left((0, T), \mathcal{D}^{\prime}(\Omega)\right) \longrightarrow L^{2}\left((0, T), \mathcal{D}^{\prime}\left(\Omega^{\prime}\right)\right) .
$$

Next, we need to prove the regularizing properties of $T_{V}$. The following result is an adaptation of [2, Prop. 4.4.].

Proposition 3.5. (Regularizing properties of $T_{V}$ ) Let $\Omega^{\prime} \Subset \Omega^{\prime \prime} \Subset \Omega$. There exists a neighborhood $V$ of the origin such that the operator $T_{V}$ defined in (3.6) has the following properties.

(1) Let $u \in \mathcal{D}^{\prime}((0, T) \times \Omega)$ such that $u=\frac{\partial^{\alpha}}{\partial x^{\alpha}} g$, for some $g \in L^{2}\left((0, T), L_{\text {loc }}^{1}(\Omega)\right)$ and multiindex $\alpha$. Then $T_{V} u \in L^{2}\left((0, T), \mathcal{D}^{\prime}\left(\Omega^{\prime}\right)\right)$ and

$$
T_{V} u=\sum_{|\beta| \leqslant|\alpha|-1} \partial_{x}^{\beta} A_{\beta} \text { in }(0, T) \times \Omega^{\prime}
$$

for suitable $A_{\beta} \in L^{2}\left((0, T), L_{\text {loc }}^{1}\left(\Omega^{\prime}\right)\right)$.

(2) Let $u \in L^{2}\left((0, T), L_{\mathrm{loc}}^{p}(\Omega)\right)$ for some $1 \leqslant p<\frac{N}{2}$, then

$$
T_{V} u \in L^{2}\left((0, T), L^{p^{\prime}}\left(\Omega^{\prime}\right)\right) \text { for } \frac{1}{p^{\prime}}>\frac{1}{p}-\frac{2}{N}
$$

and

$$
\left\|T_{V} u\right\|_{L^{2}\left((0, T), L^{p^{\prime}}\left(\Omega^{\prime}\right)\right)} \leqslant c\|u\|_{L^{2}\left((0, T), L_{\mathrm{loc}}^{p}(\Omega)\right)} \cdot
$$

(3) Let $u \in L^{2}\left((0, T), L_{\text {loc }}^{2}(\Omega)\right)$ then $T_{V} u \in L^{2}\left((0, T), W_{X}^{1,2}\left(\Omega^{\prime}\right)\right)$.

(4) Let $u \in L^{2}\left((0, T), C^{\infty}(\bar{\Omega})\right)$, then $T_{V} u \in L^{2}\left((0, T), C^{\infty}\left(\overline{\Omega^{\prime}}\right)\right)$. 
The proof of this result is a quite straightforward adaptation of the proof of [2, Prop. 4.4] and therefore will be omitted.

Corollary 3.6. Let $\Omega^{\prime} \Subset \Omega \Subset \mathbb{G}$. For every distribution $u \in \mathcal{D}^{\prime}((0, T) \times \Omega)$ such that $u=\partial_{x}^{\alpha} g$ for some multindex $\alpha$ and $g \in L^{2}\left((0, T), L_{\text {loc }}^{1}(\Omega)\right)$ there exist a neighborhood of the origin $V$ and an integer $K$ such that $\left(T_{V}\right)^{K} u \in L^{2}\left((0, T), W_{X}^{1,2}\left(\Omega^{\prime}\right)\right)$.

The proof follows exactly that of [2, Corollary 4.5].

Proposition 3.7. Let $\Omega^{\prime} \Subset \Omega$ and $V$ small enough so that $V \circ \Omega^{\prime} \Subset \Omega$. Then, for any distribution $u \in L^{2}\left((0, T), \mathcal{D}^{\prime}(\Omega)\right)$ and every left invariant operator $\mathcal{P}$ on $\mathbb{G}$ we have

$$
\mathcal{P} T_{V} u=T_{V} \mathcal{P} u \text { in } L^{2}\left((0, T), \mathcal{D}^{\prime}\left(\Omega^{\prime}\right)\right)
$$

Also, if $u \in W^{1,2}\left((0, T), \mathcal{D}^{\prime}(\Omega)\right)$ then

$$
\mathcal{L} T_{V} u=T_{V} \mathcal{L} u \text { in } L^{2}\left((0, T), \mathcal{D}^{\prime}\left(\Omega^{\prime}\right)\right)
$$

Remark 3.8. The previous proposition can be obviously iterated writing

$$
\begin{aligned}
& \mathcal{P} T_{V}^{K} u=T_{V}^{K} \mathcal{P} u \text { in } L^{2}\left((0, T), \mathcal{D}^{\prime}\left(\Omega^{\prime}\right)\right) \\
& \mathcal{L} T_{V}^{K} u=T_{V}^{K} \mathcal{L} u \text { in } L^{2}\left((0, T), \mathcal{D}^{\prime}\left(\Omega^{\prime}\right)\right)
\end{aligned}
$$

for any fixed positive integer $K$, provided $V$ is chosen small enough to have

$$
\underbrace{V \circ V \circ \cdots \circ V}_{K \text { times }} \circ \Omega^{\prime} \Subset \Omega .
$$

Proof. Let $u \in L^{2}\left((0, T), \mathcal{D}^{\prime}(\Omega)\right)$, then $T_{V} u \in L^{2}\left((0, T), \mathcal{D}^{\prime}\left(\Omega^{\prime}\right)\right)$ and for every $\varphi \in \mathcal{D}\left(\Omega^{\prime}\right)$ we can write, denoting by $\mathcal{P}^{*}$ the transpose operator of $\mathcal{P}$ and recalling that $\mathcal{P}^{*}$ is still left invariant,

$$
\begin{aligned}
\left\langle\mathcal{P} T_{V} u(t, \cdot), \varphi\right\rangle & =\left\langle T_{V} u(t, \cdot), \mathcal{P}^{*} \varphi\right\rangle=\left\langle u(t, y), \int_{\mathbb{G}} \tilde{\gamma}\left(x \circ y^{-1}\right) \mathcal{P}^{*} \varphi(x) \mathrm{d} x\right\rangle \\
& =\left\langle u(t, y), \int_{\mathbb{G}} \tilde{\gamma}(w) \mathcal{P}^{*} \varphi(w \circ y) \mathrm{d} w\right\rangle \\
& =\left\langle u(t, y), \mathcal{P}^{*} \int_{\mathbb{G}} \tilde{\gamma}(w) \varphi(w \circ y) \mathrm{d} w\right\rangle \\
& =\left\langle\mathcal{P} u(t, y), \int_{\mathbb{G}} \tilde{\gamma}(w) \varphi(w \circ y) \mathrm{d} w\right\rangle \\
& =\left\langle\mathcal{P} u(t, y), \int_{\mathbb{G}} \tilde{\gamma}\left(x \circ y^{-1}\right) \varphi(x) \mathrm{d} x\right\rangle \\
& =\left\langle T_{V} \mathcal{P} u(t, \cdot), \varphi\right\rangle .
\end{aligned}
$$

where the above equalities hold for a.e. $t$, as usual. This implies (3.7). 
To prove (3.8), it is enough to show that

$$
\partial_{t} T_{V} u=T_{V} \partial_{t} u \text { for } u \in W^{1,2}\left((0, T), \mathcal{D}^{\prime}(\Omega)\right) .
$$

Actually, for every $\psi \in \mathcal{D}(0, T)$ and $\varphi \in \mathcal{D}\left(\Omega^{\prime}\right)$ we have

$$
\begin{aligned}
\int_{0}^{T} & \psi(t)\left\langle\partial_{t} T_{V} u(t, \cdot), \varphi\right\rangle d t=\left\langle\partial_{t} T_{V} u, \varphi \otimes \psi\right\rangle \\
= & -\left\langle T_{V} u, \varphi \otimes \partial_{t} \psi\right\rangle=-\int_{0}^{T} \partial_{t} \psi(t)\left\langle T_{V} u(t, \cdot), \varphi\right\rangle \mathrm{d} t \\
= & -\int_{0}^{T} \partial_{t} \psi(t)\left\langle u(t, y), \int_{\mathbb{G}} \tilde{\gamma}\left(x \circ y^{-1}\right) \varphi(x) \mathrm{d} x\right\rangle \mathrm{d} t \\
= & -\left\langle u, T_{V}^{*} \varphi \otimes \partial_{t} \psi\right\rangle=\left\langle\partial_{t} u, T_{V}^{*} \varphi \otimes \psi\right\rangle \\
= & \int_{0}^{T} \psi(t)\left\langle\partial_{t} u(t, y), \int_{\mathbb{G}} \tilde{\gamma}\left(x \circ y^{-1}\right) \varphi(x) \mathrm{d} x\right\rangle \mathrm{d} t \\
= & \int_{0}^{T} \psi(t)\left\langle T_{V} \partial_{t} u(t, \cdot), \varphi\right\rangle \mathrm{d} t .
\end{aligned}
$$

Hence, $\partial_{t} T_{V} u=T_{V} \partial_{t} u$.

Lemma 3.9. Let $\Omega^{\prime} \Subset \Omega^{\prime \prime} \Subset \Omega$ and $u \in L^{2}\left((0, T), \mathcal{D}^{\prime}(\Omega)\right)$ satisfying the $x$-finite order assumption in $\Omega$. There exists $V$ small enough so that if

$$
T_{V} u \in L^{2}\left((0, T), C^{\infty}\left(\overline{\Omega^{\prime \prime}}\right)\right)
$$

then $u \in L^{2}\left((0, T), W_{X}^{1,2}\left(\Omega^{\prime}\right)\right)$.

The proof of this lemma is very similar to that of [2, Lemma 4.8] and therefore will be omitted.

Proof of Theorem 3.3. Fix $\Omega^{\prime} \Subset \Omega^{\prime \prime} \Subset \Omega^{\prime \prime \prime} \Subset \Omega$. By Corollary 3.6, there exist a positive integer $K$ and a neighborhood $V$ of the origin such that $T_{V}^{K} u \in L^{2}$ $\left((0, T), W_{X}^{1,2}\left(\Omega^{\prime \prime \prime}\right)\right)$. Applying Corollary 3.6 also to $\partial_{t} u$, and possibly choosing a larger integer $K$ and a smaller neighborhood $V$, we can also assume

$$
T_{V}^{K} \partial_{t} u=\partial_{t} T_{V}^{K} u \in L^{2}\left((0, T), W_{X}^{1,2}\left(\Omega^{\prime \prime \prime}\right)\right),
$$

so that

$$
T_{V}^{K} u \in W^{1,2}\left((0, T), W_{X}^{1,2}\left(\Omega^{\prime \prime \prime}\right)\right) .
$$

Let now $U \subseteq V$ a neighborhood of the origin such that

$$
\underbrace{U \circ U \circ \cdots \circ U}_{2 K \text { times }} \circ \Omega^{\prime \prime} \Subset \Omega^{\prime \prime \prime} .
$$


Let

$$
\begin{aligned}
& \Omega_{j}=\underbrace{U \circ U \circ \cdots \circ U}_{j \text { times }} \circ \Omega^{\prime \prime} \text { for } j=1,2, \ldots, 2 K \\
& \Omega_{0}=\Omega^{\prime \prime} ;
\end{aligned}
$$

so that $\Omega_{2 K} \Subset \Omega^{\prime \prime \prime}$. Clearly, it is still true that

$$
T_{U}^{K} u \in L^{2}\left((0, T), W_{X}^{1,2}\left(\Omega^{\prime \prime \prime}\right)\right)
$$

(having replaced the operator $T_{V}$ with $T_{U}$, based on a smaller neighborhood).

By Proposition 3.7 and Remark 3.8, we have

$$
\mathcal{L}\left(T_{U}^{K} u\right)=T_{U}^{K} F \text { in } L^{2}\left((0, T), \mathcal{D}^{\prime}\left(\Omega_{2 K}\right)\right) .
$$

Since, $F \in L^{2}\left((0, T), C^{\infty}(\bar{\Omega})\right)$, by point (4) in Proposition 3.5 we have $T_{U}^{K} F \in$ $L^{2}\left((0, T), C^{\infty}\left(\overline{\Omega_{2 K}}\right)\right)$. By (3.9) then $\mathcal{L}\left(T_{U}^{K} u\right) \in L^{2}\left((0, T), C^{\infty}\left(\overline{\Omega_{2 K}}\right)\right)$ and, since $T_{U}^{K} u \in W^{1,2}\left((0, T), W_{X}^{1,2}\left(\Omega_{2 K}\right)\right)$, we can apply Theorem 2.3 (iv) to conclude that $T_{U}^{K} u \in C^{0}\left([0, T], C^{\infty}\left(\overline{\Omega_{2 K-1}}\right)\right)$ and

$$
T_{U}^{K} u_{t} \in L^{2}\left((0, T), C^{\infty}\left(\overline{\Omega_{2 K-1}}\right)\right)
$$

Applying Lemma 3.9 to $u$ and $\partial_{t} u$, we see that $T_{U}^{K-1} u \in W^{1,2}\left((0, T), W_{X}^{1,2}\left(\Omega_{2 K-2}\right)\right)$. Iterating this argument $K$ times shows that $u \in W^{1,2}\left((0, T), W_{X}^{1,2}\left(\Omega^{\prime \prime}\right)\right)$. Since $F \in L^{2}\left((0, T), C^{\infty}(\bar{\Omega})\right)$ we can apply again Theorem 2.3 (iv) to conclude $u \in$ $C^{0}\left([0, T], C^{\infty}\left(\overline{\Omega^{\prime}}\right)\right)$ and $u_{t} \in L^{2}\left((0, T), C^{\infty}\left(\overline{\Omega^{\prime}}\right)\right)$.

Funding Open access funding provided by Politecnico di Milano within the CRUI-CARE Agreement.

Open Access. This article is licensed under a Creative Commons Attribution 4.0 International License, which permits use, sharing, adaptation, distribution and reproduction in any medium or format, as long as you give appropriate credit to the original author(s) and the source, provide a link to the Creative Commons licence, and indicate if changes were made. The images or other third party material in this article are included in the article's Creative Commons licence, unless indicated otherwise in a credit line to the material. If material is not included in the article's Creative Commons licence and your intended use is not permitted by statutory regulation or exceeds the permitted use, you will need to obtain permission directly from the copyright holder. To view a copy of this licence, visit http://creativecommons.org/licenses/ by/4.0/.

Publisher's Note Springer Nature remains neutral with regard to jurisdictional claims in published maps and institutional affiliations.

\section{REFERENCES}

[1] A. Bonfiglioli, E. Lanconelli, F. Uguzzoni: Stratified Lie groups and potential theory for their sub-Laplacians. Springer, Berlin, 2007. 
[2] M. Bramanti, L. Brandolini: A proof of Hörmander's theorem for sublaplacians on Carnot groups. Nonlinear Anal. 126 (2015), 170-200.

[3] M. Bramanti, L. Brandolini: Schauder estimates for parabolic nondivergence operators of Hörmander type. Journal of Differential Equations, 234 (2007), no.1, 177-245.

[4] M. Bramanti, L. Brandolini, E. Lanconelli, F. Uguzzoni: Non-divergence equations structured on Hörmander vector fields: heat kernels and Harnack inequalities. Memoirs of the AMS 204 (2010), no. 961, 1-136.

[5] M. Bramanti, M. Zhu: $L^{p}$ and Schauder estimates for nonvariational operators structured on Hörmander vector fields with drift. Analysis \& PDE 6-8 (2013), 1793-1855.

[6] F. Colombini, D. Del Santo, M. Reissig: On the optimal regularity of coefficients in hyperbolic Cauchy problems. Bull. Sci. Math. 127 (2003), no. 4, 328-347.

[7] F. Colombini, E. De Giorgi, S. Spagnolo: Sur les équations hyperboliques avec des coefficients qui ne dépendent que du temps. Ann. Scuola Norm. Sup. Pisa Cl. Sci. (4) 6 (1979), no. 3, 511-559.

[8] G. B. Folland: Subelliptic estimates and function spaces on nilpotent Lie groups. Ark. Mat. 13 (1975), no. 2, 161-207.

[9] C. Garetto, M. Ruzhansky: Hyperbolic second order equations with non-regular time dependent coefficients. Arch. Ration. Mech. Anal. 217 (2015), no. 1, 113-154.

[10] L. Hörmander: Hypoelliptic second order differential equations. Acta Math. 119 (1967), 147-171.

[11] J. J. Kohn: Pseudo-differential operators and hypoellipticity. Partial differential equations (Proc. Sympos. Pure Math., Vol. XXIII, Univ. California, Berkeley, Calif., 1971), pp. 61-69. Amer. Math. Soc., Providence, R.I., 1973.

[12] N. V. Krylov: Hörmander's theorem for parabolic equations with coefficients measurable in the time variable. SIAM J. Math. Anal. 46 (2014), no. 1, 854-870.

[13] N. V. Krylov: Hörmander's theorem for stochastic partial differential equations. Reprinted in St. Petersburg Math. J. 27 (2016), no. 3, 461-479. Algebra i Analiz 27 (2015), no. 3, 157-182.

[14] N. V. Krylov: Hypoellipticity for filtering problems of partially observable diffusion processes. Probab. Theory Related Fields 161 (2015), no. 3-4, 687-718.

[15] O. A. Oleйnik, E. V. Radkevič: Second order equations with nonnegative characteristic form. Plenum Press, New York-London, 1973.

\author{
Marco Bramanti \\ Dipartimento di Matematica \\ Politecnico di Milano \\ Via Bonardi 9 \\ 20133 Milano \\ Italy \\ E-mail:marco.bramanti@polimi.it
}

Accepted: 21 September 2020 